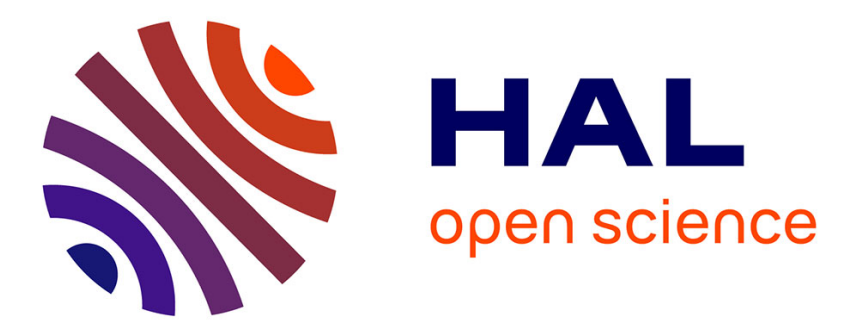

\title{
Le taux de sécurité démarchandisée: un outil d'évaluation des systèmes de protection sociale européens
}

Georges Menahem

\section{- To cite this version:}

Georges Menahem. Le taux de sécurité démarchandisée: un outil d'évaluation des systèmes de protection sociale européens. Revue Internationale de Sécurité Sociale, 2007, 2007 (2007-4), à paraître en novembre 2007. halshs-00160711

\section{HAL Id: halshs-00160711 https://shs.hal.science/halshs-00160711}

Submitted on 6 Jul 2007

HAL is a multi-disciplinary open access archive for the deposit and dissemination of scientific research documents, whether they are published or not. The documents may come from teaching and research institutions in France or abroad, or from public or private research centers.
L'archive ouverte pluridisciplinaire HAL, est destinée au dépôt et à la diffusion de documents scientifiques de niveau recherche, publiés ou non, émanant des établissements d'enseignement et de recherche français ou étrangers, des laboratoires publics ou privés. 


\title{
Le taux de sécurité démarchandisée : un outil d'évaluation des systèmes de protection sociale européens
}

\author{
Georges Menahem
}

Directeur de recherche au CNRS - CEPN-Université Paris 13 et IRDES

\section{Résumé}

Afin de mieux évaluer les rôles de la sécurité sociale et des politiques sociales dans la détermination du bien-être, cet article introduit le "taux de sécurité démarchandisée" (TSD), un instrument d'évaluation d'une mission importante de l'État social, le maintien et le développement de la sécurité économique des populations. Pour cela, il présente les conventions de son évaluation, il en analyse les principaux composants dans 20 pays européens en 2002 et il en simule les variations impliquées par une dizaine de modulations de ses composantes. L'analyse des sensibilités de la sécurité économique débouche sur la mise en évidence de trois logiques différentes. 


\section{Le taux de sécurité démarchandisée : un outil d'évaluation des systèmes de protection sociale européens}

Comment concevoir la protection sociale de demain ? Quels outils peuvent aider à penser cette protection sociale ? Nous sommes incités à nous poser ces questions à la lecture de l'article de bilan de Chantal Euzéby (2004) intitulé "Repenser la protection sociale dans l'Union européenne : vers plus de droits fondamentaux". Elle y souligne l'importance des mutations sociales auxquelles sont confrontés aujourd'hui les pays européens: les transformations du travail, ce qu'elle nomme "l'éclatement salarial", les développements associés du chômage, des précarités et des situations d'exclusion, de l'instabilité familiale et de nombreux autres facteurs tendant à mettre en cause la sécurité des individus. Elle met en rapport ce tableau alarmant avec des pans plus théoriques soulignant l'importance du souci de la sécurité : d'une part, ce qu'elle désigne comme "les principes post-welfaristes de la justice au sens de John Rawls et de Amartya Sen" ; d'autre part, la citation des déclarations de l'OIT en 1944 et des articles 22 et 25 de la Déclaration universelle des droit de l'homme de 1948 qui insiste sur la nécessité pour les individus d'une société de disposer de "sécurité économique", de "sécurité sociale" et, très précisément (dans l'article 25) de "sécurité en cas de chômage, de maladie, d'invalidité, de veuvage, de vieillesse ou dans les autres cas de perte de ses moyens de subsistance par suite de circonstances indépendantes de sa volonté".

Nous ne pouvons qu'accepter les présupposés de cet article si nous voulons approfondir le caractère central du besoin de sécurité, ce qui nous conduit à réfléchir aux moyens utiles pour "penser la protection sociale". Aussi bien pour définir ses grands principes que pour ajuster ses modalités, il apparaît essentiel de disposer d'outils: des outils théoriques, et les contributions proposées par Chantal Euzéby sont essentielles pour cela ; mais aussi des outils politiques, évidemment ; et des outils plus statistiques, enfin, pour nous permettre d'évaluer l'adéquation des moyens avec leurs fins, fins dont la sécurité des individus est un élément essentiel. Les travaux du BIT ont déjà permis d'avancer largement dans cette dernière direction. En particulier, le programme de recherche impulsé par Guy Standing (2002) afin de construire un système mondial d'enquêtes sur la sécurité des personnes est tout à fait précieux. Cette recherche ambitieuse permet de proposer la création de plusieurs indices de sécurité (du revenu, du maintien des qualifications, professionnelle, de droits syndicaux au travail, de l'emploi) et vise à prendre en compte les multiples dimensions de la vie des travailleurs, partout dans le monde.

Le propos de cet article est moins ambitieux dans la mesure où il se limite essentiellement à l'estimation des résultats des protections sociales assurées aux citoyens dans le cadre des systèmes européens de droits sociaux. Pour ce faire, nous définissons la sécurité économique des individus comme celle qui les garantit contre le risque de devenir pauvre du fait du manque de revenus, de leur perte ou de leur insuffisance. Par référence à la perspective conceptualisée par Esping-Andersen (1990) pour définir les missions de l'État de droit face au marché, nous distinguons deux types de sécurité associées à deux types de ressources : la sécurité «marchandisée » provient des revenus dits «marchandisés » parce qu'ils dépendent d'échanges marchands réalisés soit sur les marchés du travail fixant les conditions des emplois salariés, soit sur les marchés des biens et services déterminant les revenus des indépendants ou ceux de la propriété ; et la sécurité «démarchandisée » correspond aux revenus dits « démarchandisées » parce qu'ils sont indépendants des marchés et résultent soit des droits aux prestations sociales, soit d'aides et de secours versés en raison du lieu 
d'habitation ou de la situation de trop faible ressources ${ }^{1}$. La sécurité démarchandisée englobe ainsi à la fois la sécurité sociale proprement dite et les sécurités correspondant aux aides, assistances et droits sociaux assurés aux personnes démunis par les collectivités locales et les organismes d'action sociale ${ }^{2}$.

Pour paraphraser une formule du manuel sur l'État social de François-Xavier Merrien (1995), "la figure emblématique de l'État social" est dessinée par l'ensemble des droits sociaux, la garantie que la puissance publique leur apporte et ses interventions au titre de la solidarité nationale. Le présent article part ainsi des liens entre sécurité économique et développement des missions de l'État social pour proposer un outil d'évaluation de la sécurité économique assurée par les systèmes de protection sociale: le "taux de sécurité démarchandisée". Après une brève évocation du contexte historique de la confrontation de l'État social avec les forces du marché, il débouche sur la présentation de trois indicateurs visant à appréhender la sécurité économique ainsi que la "démarchandisation" des droits dont disposent les populations : le score de Esping-Andersen, un indice de sécurité canadien et le "taux de sécurité démarchandisée". L'exposé des caractéristiques de ce dernier se concrétise dans un premier tableau des composantes des sécurités associées à divers États sociaux européens. Il se conclut enfin par une estimation des multiples sensibilités du taux de sécurité démarchandisée vis-à-vis des politiques sociales.

\section{De la construction sociale de la sécurité économique des populations aux principes de son évaluation}

Aujourd'hui, dans la plupart des pays européens, la majeure partie de la population est protégée contre le risque de pauvreté. Mais cette sécurité économique n'est assurée que d'une façon plus ou moins complète, d'où le besoin de son évaluation. Cette situation est le résultat d'une longue construction sociale. L'histoire de la mise en place des missions successives de l'État social peut être lue en effet à la lumière des avatars de la sécurité matérielle des citoyens, ou encore de leur insécurité (Castel, 2003). Ainsi, au $17^{\text {ème }}$ siècle, si nous nous référons aux travaux fondateurs d'un des premiers penseurs de l'État moderne, au Léviathan de Hobbes (1651), dans l'état de nature "l'homme serait un loup pour l'homme" et l'insécurité serait totale. Pour se protéger de cette insécurité découlant nécessairement de la coexistence des hommes dans la société, il était nécessaire selon lui de développer un État fort, pourvoyeur de protections et garant de la sécurité. C'était aussi ce que disait Locke (1669) à la même époque : selon lui, l'État libéral devait avant tout protéger la propriété, laquelle assurait la sécurité de l'individu. Castel (2003) rappelle à ce propos que, jusqu'à Saint Just et Robespierre, la propriété privée est restée le socle de base fondateur de la sécurité des citoyens, lequel avait à être défendu par la République qu'ils construisaient. Ce caractère initial des États de droit ancrant la sécurité sur la propriété perdure aujourd'hui dans la structure du droit de la plupart des États industrialisés même si les fondements économiques qui en soutenaient la nécessité se sont réduits.

\footnotetext{
1 Nous ne prenons donc pas en compte dans cet article les sécurités économiques garantissant contre certains risques évoqués notamment par Ulrich Beck (2000) dans La société du risque et qui concernent les éventualités de catastrophes naturelles ou industrielles ou qui sont associées aux possibilités de troubles terroristes, délictueux, criminels ou de guerre, risques relevant davantage de la société civile.

2 Si nous faisons référence à la définition de la protection sociale proposée par Karl Polanyi (1944) qui, selon lui, regroupe trois grandes modalités d'allocation (l'assurance privée, la solidarité familiale et la redistribution publique et sociale) afin de protéger les travailleurs face à la logique de marché, nous pouvons noter que notre approche de la sécurité démarchandisée ne concerne que le troisième terme de la protection sociale selon cet auteur.
} 


\section{L'État garant de ressources et de sécurités démarchandisées}

$\mathrm{Au}$ cours des deux derniers siècles, cette mission de garant de la sécurité assurée par l'État social s'est largement développée en opposition aux pressions déstructurantes du marché, même si l'avènement du salariat a transformé fortement les bases de la sécurité en ruinant dans les pays industrialisés une grande partie des petites propriétés marchandes. À mesure que les conquêtes sociales ajouteront des garanties en cas d'accident de travail, puis de maladies professionnelles, puis de retraite et de chômage, la situation de salarié sera associée à un nombre de plus en plus important de sécurités. Ainsi, dans l'Allemagne de Bismarck puis en France et au Royaume-Uni, les premières institutions de protection sociale vont progressivement consolider ces sécurités partielles jusqu'à leur intégration dans les droits d'une sécurité sociale légitimée par l'État, puis dans l'édification du statut juridique de "salarié". L'arrivée au pouvoir de coalitions portée par les syndicats dans les régimes sociauxdémocrates du Nord de l'Europe, enfin, développera les caractères d'une société salariale dans laquelle le statut de salarié permet l'acquisition de sécurités tout au long de la vie. Si, dans les dernières décennies, les dérégulations des marchés des capitaux et la fragilisation des cadres du travail se sont traduites dans la remise en cause de nombreuses protections associées au salariat, il n'en reste pas moins que l'État social garantit encore aujourd'hui une grande part des sécurités des populations dans les pays développés. Parmi les protections que l'État social a pour mission de garantir, la sécurité économique, qui protège contre le risque de ne pas disposer de revenus suffisants, tient une place essentielle.

Une des premières insécurités des populations résulte du caractère variable de leurs revenus et de leurs autres ressources économiques. L'instabilité des activités marchandes et des ressources qu'elles procurent s'ajoute aux accidents aléatoires de la vie pour mettre fréquemment en cause les capacités des personnes à disposer régulièrement de moyens pour se nourrir, se loger, se vêtir et subvenir à leurs besoins fondamentaux. Pour échapper aux risques de pauvreté, il est essentiel de disposer des protections que fournissent les systèmes de sécurité sociale et les aides garanties par la puissance publique. Elles sont d'autant plus précieuses que leur disposition est inconditionnelle et n'est pas soumise aux aléas des commandes ou de la conjoncture des marchés. Dans ce sens, elles sont "démarchandisées », pour reprendre le concept de Polanyi (1944) tel qu'il a été développé et adapté aux nouveaux caractères des États sociaux à travers les travaux de Esping-Andersen (1990).

Ce sociologue suédois, afin d'ordonner et interpréter la profusion des systèmes de protection sociale dans son livre Les trois mondes de l'État providence, a centré son analyse des États modernes autour du concept de «démarchandisation ». Il a défini ainsi cette notion : «La démarchandisation survient lorsqu'un service est obtenu comme un dû et lorsqu'une personne peut conserver ses moyens d'existence sans dépendre du marché $»^{3}$. Une telle indépendance distingue fortement les ressources ainsi « démarchandisées » du cas général de soumission à la logique du marché, ce qu'il a caractérisé ainsi : "Quand la force de travail devient une marchandise, la possibilité pour les individus de survivre hors du marché est mise en jeu. Ceci constitue le problème essentiel et conflictuel de la politique sociale. » $[\ldots]$ « Le bien-être et la survie en viennent à dépendre de la bonne volonté d'une personne à engager une force de travail $»^{4}$. L'essentiel ici est de souligner en quoi ce concept amène à distinguer clairement des sécurités marchandisées, qui sont soumises aux aléas du marché et des décisions patronales, et des sécurités démarchandisées qui, elles, relèvent du contrôle de l'État et des autorités publiques. Par référence aux travaux de Orloff (1993) et Jane Lewis (1992) soulignant la portée de la démarchandisation pour la transformation des rapports de genre,

\footnotetext{
3 Esping-Andersen (1990, trad. fr. 1999, p.35).

${ }^{4}$ Ibidem, pages 51 et 52 .
} 
nous pouvons remarquer que les sécurités démarchandisées sont aussi « défamilialisées » et permettent aux femmes d'être moins dépendantes de la présence de solidarités familiales ou conjugales, dans la mesure de l'existence de comptes et de droits individuels pour les femmes. Il serait possible de complexifier encore cette analyse en introduisant le jeu des autres acteurs sociaux comme nous incitent à le faire les analyses présentées par Merrien (2005) ${ }^{5}$. En suivant les travaux mis en avant par ce dernier, on peut en effet se demander si les ressources et la sécurité « démarchandisées » ne deviennent pas des biens sociaux appropriés à la fois par l'État, par les syndicats et les organisations patronales en fonction de compromis sociaux dépendant étroitement des histoires et spécificités de chaque pays.

\section{Deux évaluations de la sécurité démarchandisée}

Le développement de nombreuses institutions publiques contribuant à « démarchandiser » la sécurité rend de plus en plus utile la disposition de concepts et d'outils statistiques permettant d'évaluer les conséquences des politiques sociales en matière de sécurité ou d'insécurité des populations. Afin d'affiner sa typologie et d'illustrer sa pertinence, EspingAndersen (1990) a élaboré un outil statistique qu'il a nommé indicateur de démarchandisation. Cet indicateur a été conçu avant tout à partir d'une analyse théorique visant à distinguer nettement trois ensembles de pays et a permis d'illustrer le bien-fondé de la typologie des systèmes de protection sociale. Peut-il être utilisé pour d'autres objectifs que la mission pour laquelle il a été construit ? Il agrège en effet des variables de statuts très divers, à la fois qualitatives et quantitatives, et qui concernent trois domaines très différents ${ }^{6}$. Or les conventions d'évaluation des trois scores dont il fait la moyenne étant inconnues, il est impossible de le reproduire pour le tester sur d'autres dates ou d'autres pays. Il n'est pas possible a fortiori de l'utiliser pour simuler les effets des modulations des politiques sociales, que ce soit sur la sécurité ressentie des personnes ou sur leur bien-être.

Une autre tentative découle des travaux de deux chercheurs canadiens qui ont élaboré un indicateur de bien-être économique dont un objectif essentiel est de montrer en quoi le PIB par habitant procure une mesure du bien-être économique largement insatisfaisante (Osberg et Sharpe, 2002). Son principe consiste à faire la moyenne de quatre indicateurs synthétiques : le premier porte sur les flux de consommation au sens large; le deuxième intègre les considérations de patrimoine économique et humain et cherche à prendre en compte les dégats environnementaux; le troisième agrège des indicateurs synthétiques d'inégalités avec des indices de pauvreté économique relative; le quatrième enfin évalue quatre types d'insécurité économique liés respectivement au chômage, à la maladie, à la vieillesse, et à la situation des familles monoparentales.

Pour mesurer l'insécurité, ces auteurs ont fait le choix d'évaluer le risque subjectif d'anxiété des individus à partir de quatre situations dont ils pouvaient évaluer schématiquement le coût moyen pour les individus concernés, ce qui les obligeait à faire quelques approximations et une hypothèse économique qu'ils ont formulée ainsi : "le changement de risque subjectif est supposé être proportionnel aux variations de risque objectif"7. Le coût de la maladie a ainsi été estimé par les seules dépenses médicales non remboursées (ce qui laisse de côté le manque

\footnotetext{
5 Opus cité, chapitre 4, pp. 37-43.

${ }^{6}$ Les unes sont juridiques (les règles d'éligibilité à la capacité à avoir droit à des ressources), les autres institutionnelles (les conditions de durée de cotisation, la durée des droits garantis et la période d'attente nécessaire pour obtenir un tel revenu) et les dernières économiques (le niveau des revenus de remplacement et la part individuelle du financement des prestations) (opus cité, trad. fr., 1999, pp. 73-74. Il faut préciser que l'appendice du livre décrivant cet indicateur ne comporte ni formule ni étapes de calcul, qu'il est donc difficile de reproduire l'indice de démarchandisation et impossible de calculer les parts de chaque variable.

7 Osberg et Sharpe (2002), page 305.
} 
à gagner consécutif aux arrêts de travail); celui des pertes d'emploi a été obtenu à partir du taux de remplacement des indemnités de chômage ; celui du divorce et celui de la vieillesse à partir du taux de pauvreté moyen des mères seules et des personnes âgées ${ }^{8}$. Ces calculs ont finalement l'avantage de procurer des estimations réalistes de l'importance des insécurités associées à quatre types de risque économique. D'où la possibilité de modéliser, moyennant des approximations supplémentaires ${ }^{9}$, les variations de sécurité économique des individus, opération qui n'était pas permise par l'indice de démarchandisation de Esping-Andersen.

Mais les évaluations des insécurités ainsi obtenues ne fournissent que des approximations très partielles des pertes de revenus impliquées par la maladie, le chômage, le divorce ou la retraite. Par conséquent, seules les variations de l'insécurité au cours du temps peuvent être évaluées dans chacun des pays. Il n'est donc pas possible de comparer les niveaux respectifs de la sécurité économique entre les différents pays occidentaux. De plus, toute analyse de l'insécurité devient très difficile puisque l'on ne peut comparer valablement le risque induit par le chômage à celui de la maladie, du divorce ou de la vieillesse. Là encore, il n'est pas possible d'utiliser cet autre indicateur pour simuler les intérêts des différentes modulations des politiques sociales.

\section{Les principes et conventions fondant l'évaluation $d u$ "taux de sécurité démarchandisée »}

Partant d'une approche directement économique et globale, notre projet a été de dépasser les limites des indicateurs précédents afin $1^{\circ}$ de pouvoir comparer les performances relatives des différents États sociaux en matière de lutte contre l'insécurité économique des populations, $2^{\circ}$ d'analyser les rôles des différents facteurs de risque et $3^{\circ}$ de simuler l'action des diverses politiques sociales sur la sécurité. Comme le rappelle Jean Gadrey et Florence Jany-Catrice (2003, p. 73) en conclusion d'un article de bilan des indicateurs alternatifs du développement et du progrès social, "on voit mal comment se passer de 'conventions discutables' dès lors qu'il est question d'environnement, de qualité de vie et de progrès social, et donc de systèmes de valeurs. Même les indicateurs économiques 'sérieux' sont truffés de conventions discutables, comme l'ont montré les débats sur les incertitudes très importantes des comparaisons du PIB par habitant en Europe". Esping-Andersen a privilégié ainsi un point de vue institutionnel pour évaluer ce qu'il a appelé «le potentiel de démarchandisation des politiques sociales ${ }^{10}$. De notre côté, pour évaluer la sécurité des populations, nous avons décidé de partir d'un point de vue plus individuel en ce qui concerne l'appréciation du niveau des ressources et, en particulier, pour estimer le niveau où commence l'insécurité économique. Il va alors de soi que ces deux points de vue différents ont entraîné la construction de deux types différents d'indicateurs. Les conventions que nous avons adoptées (cf. Menahem et Cherilova, 2005) doivent être précisées du fait de leur caractère fortement normatif. Elles sont listées ci-après et définissent pour l'essentiel trois grands principes :

Les ressources concernées: nous avons décidé d'estimer, en tant que ressources d'une sécurité économique indépendante des liens avec les employeurs, les revenus démarchandisés qui proviennent des systèmes de protection sociale ou d'aide publique et que les personnes

\footnotetext{
8 Ibidem, pages 306-308.

${ }^{9}$ Ces approximations non explicitées dans l'article cité doivent permettre de considérer que la probabilité du risque total d'insécurité est égale à la somme des probabilités des 4 risques pris en compte. Pour l'accepter il faut supposer que les variations des probabilités partielles sont additives (négatives pour l'insécurité et positives pour la sécurité) et donc $1^{\circ}$ que leur importance est marginale et $2^{\circ}$ qu'elles sont mutuellement indépendantes.

${ }^{10} \hat{A}$ savoir "les règles gouvernant les accès aux droits, les critères reconnus d'éligibilité et le niveau de remplacement du revenu autorisé par les diverses prestations", cf. opus cité, page 66.
} 
perçoivent aux différents âges de leur vie adulte. Une telle donnée permet en effet d'apprécier quelle est la part des individus qui ne disposent pas de revenus suffisants pour assurer de nombreuses sécurités primordiales telles que la capacité à se nourrir, se loger ou se vêtir. Ces revenus sont de trois types :

- les revenus de remplacement du travail associés aux risques vieillesse, chômage, maladie, invalidité ainsi que les pensions de réversion (veuvage),

- les remboursements et aides en nature : frais de maladie, charges familiales, aides au logement,

- les indemnités et aides en nature mobilisées dans la lutte contre l'exclusion sociale (RMI, etc.).

La base de comparaison: nous avons évalué pour chaque pays ou territoire les ressources contribuant à la sécurité relativement au niveau moyen du revenu disponible des actifs ayant un emploi dans le lieu considéré. Ce principe est essentiel dans la mesure où il s'appuie sur l'hypothèse que les habitants d'un territoire donné évaluent leur niveau de vie relativement à celui des personnes considérées comme «standards» dans le système médiatique et institutionnel de référence, à savoir celles vivant dans leur environnement social et ayant un emploi (référence que nous approchons par le revenu disponible moyen des actifs en emploi, soit précisément par leur revenu net d'impôts et de cotisations, et comprenant les prestations famille, logement, maladie). Le "taux brut des sécurités économiques démarchandisées" (TBSD) qui en résulte est égal au rapport entre la somme des revenus démarchandisés et le revenu de référence du territoire considéré.

La pénalisation de l'insécurité économique: Afin de prendre en compte la première des insécurités économiques, à savoir celle des personnes affrontant des situations de pauvreté du fait de leurs trop faibles revenus, nous avons considéré que les ménages dont les ressources par tête ne dépassent pas le seuil de pauvreté dans le territoire considéré ont un niveau de sécurité économique égal à zéro et que leur situation est pénalisée par une insécurité égale à la différence entre leurs revenus et le revenu de référence. Pratiquement, l'indicateur pondère négativement la population en dessous du seuil de pauvreté en lui affectant un revenu négatif dont le montant est égal à $60 \%$ du revenu disponible moyen des personnes ayant un emploi ${ }^{11}$. Cette forte pénalisation veut rendre compte du point de vue des populations sans ressources dont l'insécurité économique lamine les capacités de bien-être et de survie. Elle signale aussi la mise en cause des capacités des institutions ayant pour mission d'assurer aux individus une sécurité indépendante des aléas du marché. Au niveau global, elle doit donc être déduite de la sécurité démarchandisée procurée par les diverses institutions composant l'État social.

Ce dernier principe manifeste l'importance que nous avons voulu donner à l'objectif de cohésion sociale, laquelle constitue, selon notre point de vue, un des éléments importants de la sécurité d'une population vivant dans un territoire donné. Au total, le taux de sécurité économique démarchandisée (TSD) est un indicateur à la fois synthétique et relatif.

\footnotetext{
${ }^{11}$ Cette évaluation de l'insécurité économique est voisine du seuil de pauvreté, soit $60 \%$ du revenu médian par unité de consommation après prestations dans la définition de Eurostat, ce qui explique l'adoption de ce niveau de pondération qui permet d'avoir une pénalisation cohérente avec le revenu de référence adopté au dénominateur.

Nous pouvons noter de plus que la discontinuité dans la courbe du TSD en fonction du revenu des personnes est atténuée par l'existence d'une part non négligeable de personnes disposant d'un revenu proche du seuil de pauvreté. Étant donné que la sécurité économique est évaluée par la différence entre leurs revenus et 0,6 fois le revenu de référence, après une chute à zéro quand le revenu atteint le seuil de pauvreté, la courbe diminue lentement jusqu'à sa valeur minimum, $-0,6^{*}$ Revenu de référence, quand le revenu continue à décroitre.
} 
Synthétique, il fait la synthèse entre un ratio brut concernant le niveau des ressources démarchandisées et le niveau de pauvreté relative. Relatif, il fait la somme des revenus issus de droits sociaux que les individus touchent tout au long de leur vie adulte dans le pays considéré et les divise par un dénominateur représentant le niveau de vie moyen des actifs ayant un emploi, déduction faite de l'insécurité relative subie par les ménages en situation de pauvreté.

Les formules de calcul du TSD : L'ensemble de ces considérations de méthode se traduit dans des formules comptables permettant de calculer et comparer les niveaux de sécurité démarchandisée des diverses populations. Nous pouvons les présenter sous deux formes plus ou moins agrégées. D'abord, à un niveau très macroéconomique, si nous appelons TSD le taux de sécurité démarchandisée et TIE le taux d'insécurité économique associée aux personnes vivant dans des ménages en dessous du seuil de pauvreté, nous avons :

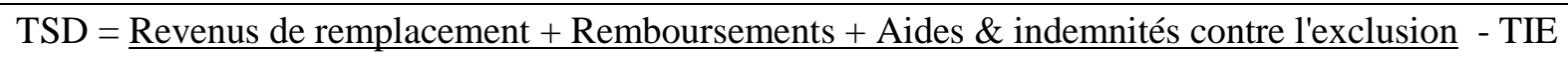
Population adulte $*$ Revenu disponible moyen des actifs ayant un emploi

Avec TIE $=\underline{\text { Population pauvre }} * \underline{60 \% * \text { Revenu disponible moyen actifs employés }}$ Population adulte $\quad$ Revenu disponible moyen actifs employés

Une deuxième formule résultant d'une approche partiellement désagrégée peut également être présentée. Il est en effet possible de décomposer la population adulte en trois catégories selon le type des revenus démarchandisés perçus : les individus qui touchent des revenus de remplacement (indemnités de chômage, d'invalidité, retraites et pensions de réversion) ; ceux qui touchent des remboursements et aides en nature correspondant à leurs soins de santé ou des aides au logement; et ceux qui touchent les aides et indemnités de lutte contre l'exclusion (minimums sociaux du type RMI, minimum vieillesse et autres indemnités dites "de solidarité"). Il faut préciser que les recouvrements entre les quatre populations ici artificiellement distinguées peuvent être importants : par exemple, certains chômeurs et retraités pauvres peuvent d'un coté toucher des allocations retraites ou chômage, d'un autre coté percevoir des remboursements de soins ou des aides au logement et, leurs revenus étant trop faibles pour les sortir de la pauvreté, participer à la pénalisation du TSD induite par la dernière ligne négative de la formule. Sachant que chaque population doit être pondérée par son importance statistique et qu'il faut ajouter à ces revenus démarchandisés directs d'autres revenus indirects correspondant aux remboursements des frais de maladie ou autres, nous avons alors la deuxième formule suivante ${ }^{12}$ :

$$
\begin{aligned}
\mathrm{TSD}= & \% \text { Retraités, chômeurs, invalides } * \frac{\text { Revenu moyen de remplacement (retraite, chômage) }}{\text { Revenu disponible moyen. actifs employés }} \\
& +\frac{\text { Montant moyen des remboursements de soins \& aides en nature et au logement }}{\text { Revenu disponible moyen. actifs employés }}
\end{aligned}
$$

$+\%$ Pauvres \& Exclus * Aides \& indemnités contre l'exclusion \& minimum vieillesse Revenu disponible moyen actifs employés

- $0,6 * \%$ Population pauvre

12 Pour effectuer le passage d'une formule à l'autre, il suffit de remarquer que chaque montant global de revenu est égal au produit de la population concernée (soit le \% dans la population adulte*population adulte) par le revenu moyen correspondant. Par exemple pour les allocations de retraite on a : Montant global de retraite $=\%$ retraité parmi les adultes $*$ Population adulte * Montant moyen de retraite par retraité. Quand on divise chacun de ces termes par le dénominateur, on simplifie en enlevant la population adulte au numérateur et dénominateur, et on obtient: \% retraités * Montant moyen de la retraite / revenu disponible moyen des actifs en emploi. 
Pour examiner en quoi les concrétisations de ces formules théoriques permettent de rendre compte de la réalité des différences de sécurité entre les pays, nous avons eu recours aux bases de données conçues et rassemblées par Eurostat. Par là, nous avons hérité des catégories et nomenclatures que cet organisme européen a élaborées, en particulier à propos des prestations sociales qu'il a séparées en huit groupes dans son système dit SYSPROS (voir en annexe 1).

\section{Les caractères de la sécurité démarchandisée dans 20 pays européens}

Les données d'Eurostat disponibles fin août 2006 nous ont permis d'évaluer le taux de sécurité démarchandisée dans 20 pays européens pour l'année $2002^{13}$. Ces estimations présentées dans le graphique 1 nous permettent d'observer des différences considérables entre les niveaux de sécurité des populations européennes, d'où la mise en évidence de quatre groupes de pays homogènes en matière de garantie économique. La décomposition des sources de la sécurité économique illustrée dans le tableau 1 et représentée dans le graphique 2 nous permettra de mieux comprendre les origines de ces disparités qu'il est possible de rattacher aux histoires institutionnelles de ces pays. Puis l'exposé rapide des limites de nos estimations nous conduira à en relativiser les conclusions.

Quatre principaux groupes de pays européens du point de vue de la sécurité démarchandisée

La grande diversité des résultats des systèmes européens de prestations sociales apparaît clairement dans le graphique 1. Une opposition historique s'y dessine entre, d'une part, les pays du Nord, les pays continentaux et le Royaume-Uni dont les systèmes de protection sociale se sont développés fortement depuis plus d'un demi-siècle et, d'autre part, les pays du Sud et les PECO dont l'organisation et les systèmes de protection restent sommaires ou sont bien plus récents. Dans le premier grand groupe, la sécurité démarchandisée varie entre $13 \%$ et $28 \%$ du revenu disponible moyen des actifs ayant un emploi alors que, dans le deuxième grand groupe, elle ne varie qu'entre $3 \%$ et $11 \%$. Mais le deuxième groupe est loin d'être homogène et nous pouvons y distinguer trois sous-groupes bien distincts.

\footnotetext{
${ }^{13}$ Le grand avantage des données d'Eurostat est qu'elles sont fournies dans un cadre cohérent qui permet des comparaisons internationales. Mais, comme elles sont fournies par les administrations des différents pays européens, elles ne sont disponibles qu'avec des rythmes et des contraintes particulières. Ceci fait que la table que nous avons constituée n'a permis de rassembler les différentes variables nécessaires au calcul du TSD pour les années 1995 à 2002 que pour 20 pays. Nous pouvons noter que ce nombre est supérieur de 7 à celui que nous avions obtenu pour les années 1995 à 2000 sur les données de 2004. Voir en annexe
} 


\section{Graphique 1 : Les niveaux du taux de sécurité démarchandisée} dans 20 pays européens en 2002

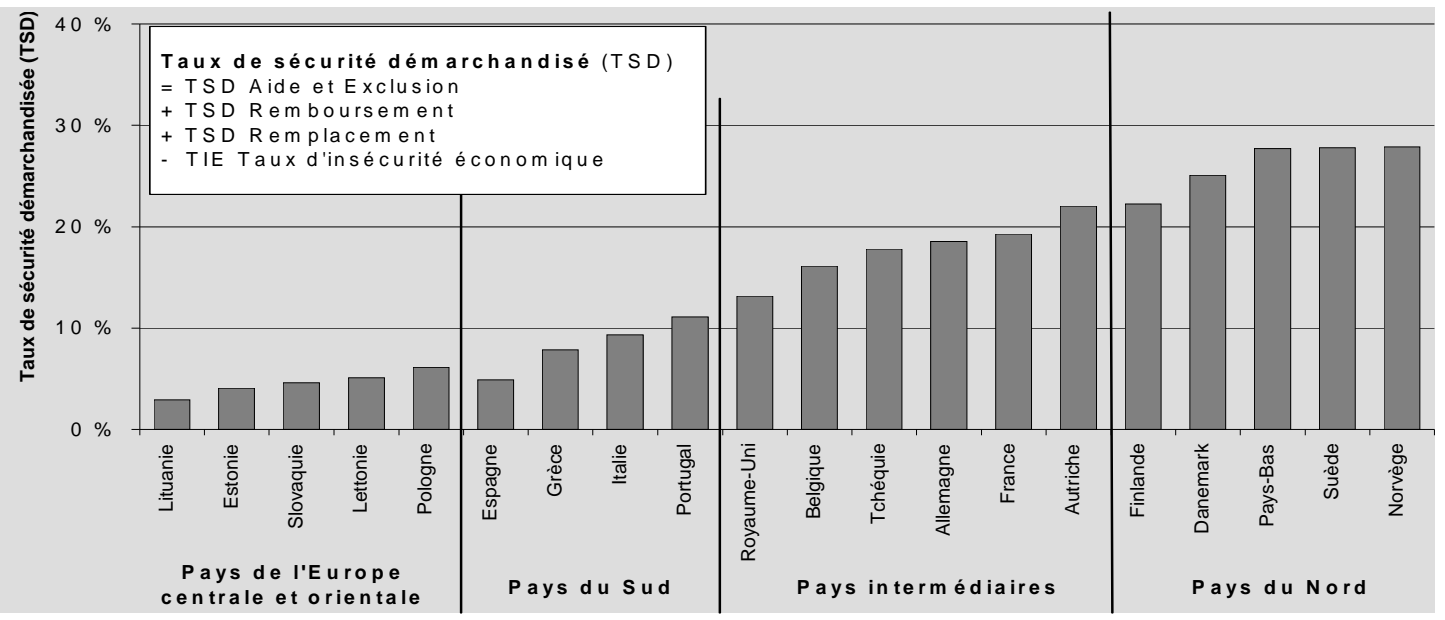

Source : Eurostat 2006

Lecture : le TSD net de l'insécurité de 2,9\% de la Lituanie est égal à la différence entre ses faibles ressources de sécurité $(13,1 \%)$ et sa forte insécurité économique (-10,2\%). Au Royaume-Uni, la forte insécurité (- 10,8\%) est compensée par d'importantes ressources de sécurité (23,9\%), d'où un TSD de $13,1 \%$, quatre fois supérieur à celui de la Lithuanie.

Graphique 2 : Les trois grandes ressources de sécurité et l'insécurité économique entrant dans le taux de sécurité démarchandisée dans 20 pays européens en 2002

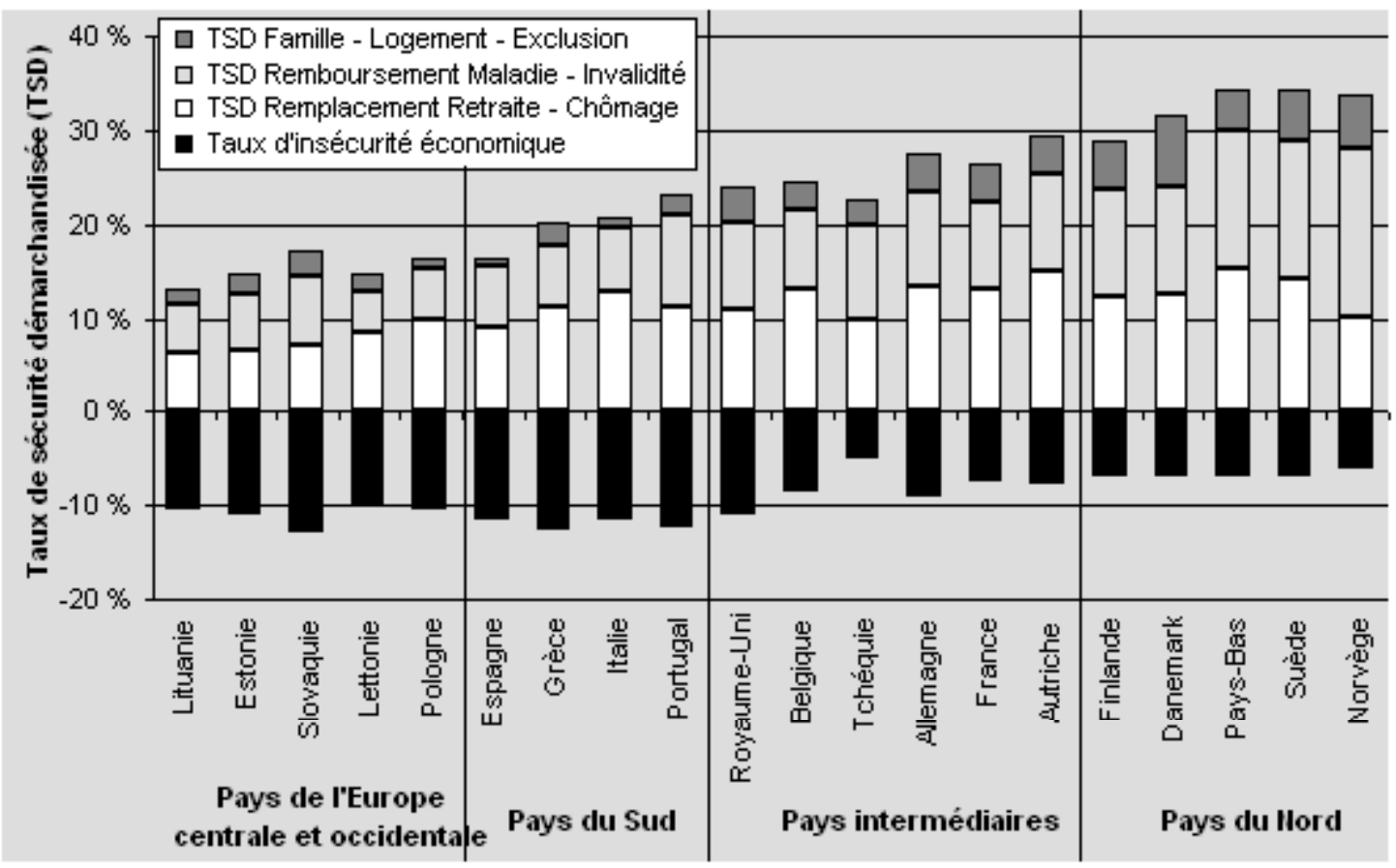

Source : Eurostat 2006

Lecture : Ce deuxième graphique explicite les éléments positifs et négatifs entrant dans la composition de la sécurité démarchandisée. Ainsi, en Espagne en 2002, le TSD n'est que de 4,9\% du revenu disponible moyen des actifs ayant un emploi (soit la somme des sécurités retraite, chômage 9,2\%, maladie et invalidité 6,4\%, aide aux familles et aux démunis 0,7\%, moins l'insécurité 11,4\%). En revanche, elle s'élève en Norvège à $27,9 \%$ du revenu de référence moyen, soit cinq fois plus à la même date (avec respectivement les sécurités 10,2\%+17,9\%+5,8\%, moins l'insécurité $6 \%$ ). 
Nous pouvons remarquer à ce propos que la typologie associée au taux de sécurité démarchandisée est en grande partie compatible avec celle proposée par Esping-Andersen (1990). Le groupe des pays du Nord est pour l'essentiel équivalent au groupe des pays dits du « régime socialiste » de Esping-Andersen et le groupe des pays intitulés «intermédiaires »se rapproche beaucoup du régime dit «conservateur », sauf deux notables exceptions sur dix pays : l'Italie classée dans le régime conservateur n'est pas rattachée au groupe dit des intermédiaires; le Royaume-Uni, lui, y est associé alors qu'il est classé dans le régime «libéral $»^{14}$. Le fait que nos estimations aient concerné uniquement des pays européens et que Esping-Andersen n'ait pris en compte ni d'autres pays du Sud ni les PECO empêche d'aller plus loin dans la comparaison.

Ainsi, les cinq "pays du Nord" de TSD maximum se rattachent à la même tradition socialedémocrate d'intervention publique forte, de prestations généreuses et de couverture universelle, même s'ils n'ont pas affronté la crise des années 90 avec les mêmes réformes ${ }^{15}$. Le niveau de leur TSD témoigne des proximités de leur type d'État social : il varie en 2002 entre $22,2 \%$ et $27,9 \%$. En revanche, le regroupement des dits "pays intermédiaires" est beaucoup plus hétérogène puisqu'il agrège un ancien pays socialiste avancé, la République Tchèque, avec quatre États dits "conservateurs" selon la typologie de Esping-Andersen (1990) (la Belgique, l'Allemagne, la France et l'Autriche) et un pays dit "libéral" (le Royaume-Uni). Leurs TSD sont un peu plus dispersés puisqu'ils s'échelonnent entre 13,1\% pour le RoyaumeUni et $22 \%$ pour l'Autriche.

Le groupe des quatre pays dits "du Sud" rassemble de nombreux caractères communs. La faiblesse du développement de l'État social s'y est conjuguée longtemps avec la présence de traditions clientéliste et corporatiste, d'où des formes variables d'obstacles aux réformes. La Grèce, l'Italie, l'Espagne et le Portugal combinent ainsi chacun d'une façon particulière le développement des transferts financiers sur des bases corporatistes, la faiblesse des aides et services aux familles et démunis, et le développement de réseaux familiaux pour compenser ces insuffisances ${ }^{16}$. Il est frappant de constater que, au-delà de cette variété, ces quatre pays se retrouvent regroupés dans le partage d'une même faiblesse de la sécurité démarchandisée : entre 4,9\% pour l'Espagne et $11,1 \%$ pour le Portugal.

D'où vient l'écart important du TSD entre l'Espagne et le Portugal ? Il s'explique en partie par les formes différentes de la mobilisation de la force de travail qui s'y sont établies : elles se traduisent d'abord dans un taux d'emploi bien plus important au Portugal (49,3\% de la population totale en 2002) qu'en Espagne (respectivement 39,4\%), ce qui est associé avec une plus grande capacité du système social portugais à percevoir des cotisations et à assurer un haut niveau de prestations démarchandisées. Autre particularité, l'engagement des femmes dans l'activité professionnelle bien plus important au Portugal (46\% de la population active en 2002) qu'en Espagne (40\%). Ces particularités impliquent un engagement plus important

\footnotetext{
${ }^{14}$ Dans la typologie de Esping-Andersen (1990, p. 70), l'Italie a en effet un «indice de démarchandisation » de 24,1 , ce qui l'incite à placer ce pays dans le régime «conservateur », devant le Royaume-Uni situé à 23,4, placé lui dans le régime dit «libéral». Selon le TSD, les variables institutionnelles historiques sont moins privilégiées et l'ensemble des ressources démarchandisées est le seul critère objectif de classement. Ceci explique que le TSD du Royaume-Uni soit de 13,1\%, nettement devant l'Italie, à 9,3\%, et que le RoyaumeUni relève du groupe des pays intermédiaires et l'Italie du groupe des pays du Sud (Menahem, 2006, p. 328).

${ }^{15}$ Voir le bilan complet des transformations de "l'État providence nordique" dans le numéro spécial de la Revue française des affaires sociales présenté par Pierre Strobel (2003) et regroupant les interventions à un colloque organisée par la MIRE.

${ }^{16}$ Voir l'autre ensemble de textes rédigés à l'occasion des "Rencontres de Florence" organisée par la MIRE pour "Comparer les systèmes de protection sociale en Europe du Sud" et conclu par Rhodes et Palier (1997).
} 
de l'État social pour assurer de façon démarchandisée les soins aux enfants et aux personnes âgées au Portugal, là où les femmes sont plus professionnellement actives.

Le groupe dit "des PECO" (pays d'Europe centrale et orientale) présente aussi une homogénéité historique. Les cinq États sociaux correspondants sont tous issus d'un même système socialiste, ont engagé depuis des dates variables un même processus de transition vers l'économie de marché et se sont largement intégrés dans l'Union européenne. Bien que les histoires des définitions de leurs systèmes sociaux soient, elles aussi, spécifiques, il est aussi remarquable de constater la proximité de leurs bas niveaux de TSD : entre 2,9\% pour la Lituanie et $6,1 \%$ pour la Pologne. Deux questions se posent à propos du bas niveau des TSD dans les PECO : comment se fait-il qu'il soit si faible alors qu'avant 1989, les États socialistes de ces pays assuraient à toute la population un niveau minimum de sécurité ? Et pourquoi le niveau du TSD de la Tchéquie leur est-il supérieur d'au moins 11\% alors que ce pays partage de nombreux caractères historiques avec les autres PECO ? Tout d'abord, le transfert du financement des retraites, des soins de santé et des principales prestations sociales, naguère assurés par l'État socialiste dans les PECO, à un système fondé sur des cotisations sociales n'a débuté qu'au plus depuis une quinzaine d'années. Or il s'agit d'un processus qui nécessite de profondes réorganisations et qui prendra beaucoup de temps. Ce retard s'est trouvé accentué par un phénomène de fuite devant l'impôt entraîné par la hausse des cotisations pesant sur les entreprises et les particuliers et par le fait que les entreprises ont renoncé à assurer un certain nombre de prestations sociales. Quant au meilleur TSD de la Tchéquie, il est dû pour l'essentiel au développement bien plus important dans ce pays de «caisses de santé » finançant de manière décentralisée le système de santé et à la croissance économique plus vigoureuse après 1994 qui s'est traduit dans une réduction plus importante de la pauvreté.

\section{Les liens entre les caractères des trois principales composantes de la sécurité démarchandisée et l'insécurité économique}

L'analyse de la structure du TSD est chiffrée dans le tableau 1 et représentée dans le graphique 2. Elle permet de formuler quatre constats rapides : les trois premiers caractérisent les ressources démarchandisées représentées en positif au-dessus de l'axe et variant entre $13 \%$ et $34 \%$; le dernier concerne les niveaux de l'insécurité économique, en négatif audessous de l'axe, allant de $5 \%$ à $13 \%$.

1. Les revenus de remplacement de l'activité professionnelle varient faiblement. Ils sont composés des retraites, des pensions de réversion, des indemnités de chômage et d'aide à l'insertion dans l'activité. Leur importance obéit à la fois à des traditions historiques, à des conjonctures locales, et à la capacité des syndicats, du patronat et de l'État à établir des compromis sociaux. Avec les difficultés de mise en place de nouveaux systèmes de cotisation sociale, ceci explique que ces sécurités soient encore peu importantes dans les États sociaux récemment formés des PECO : 10\% au maximum comme en Pologne et en République tchèque. En revanche, dans les pays de traditions corporatistes anciennes ces sécurités démarchandisées atteignent des sommets, comme en Autriche et aux Pays-Bas (15\%), en Suède et en Allemagne (14 \%), ou encore en France, en Italie et au Danemark (13\%). Mais il faut en même temps souligner les différences qui peuvent résider derrière de telles proximités, par exemple entre la recherche d'activation danoise et les traditions corporatistes italiennes.

2. Les revenus issus des remboursements des dépenses de maladie et d'invalidité varient plus fortement. Les traditions d'un large financement public du système de santé et de prise en charge généreuse de l'invalidité dans les pays nordiques se distinguent de la fragilité des systèmes en voie d'édification dans les PECO. Les premiers consacrent de $11 \%$ à $18 \%$ à de 
telles dépenses alors que les seconds restent limités entre $4 \%$ et $8 \%$. Entre ces extrêmes, les Pays du Sud, le Royaume-Uni et les pays continentaux balancent entre la générosité et la voie des réformes visant à compresser les coûts du système de santé.

3. Les aides à la famille et aux démunis sont les plus variables. Elles s'élèvent à près de $8 \%$ en Norvège alors qu'elles n'atteignent pas $1 \%$ en Pologne. Dans les pays du Sud et dans les PECO, là où ces sécurités ne dépassent pas $2,5 \%$, des réseaux familiaux compensent évidemment le défaut des politiques publiques d'assistance. Mais ils procurent des sécurités plus incertaines parce que dépendantes des relations personnelles.

Tableau 1 - Les quatre composantes du taux de sécurité démarchandisée dans 20 pays européens en 2002

\begin{tabular}{|c|c|c|c|c|c|}
\hline \multirow[b]{2}{*}{ Pays du Nord } & \multirow[t]{2}{*}{$\begin{array}{c}\text { Taux } \\
\text { d'insécurité } \\
\text { économique }\end{array}$} & $\begin{array}{c}\text { Remplacement } \\
\text { Retraite } \\
\text { Chômage }\end{array}$ & \multirow[t]{2}{*}{$\begin{array}{c}\text { Remboursement } \\
\text { Maladie } \\
\text { invalidité }\end{array}$} & \multirow[t]{2}{*}{$\begin{array}{l}\text { Famille } \\
\text { Logement } \\
\text { Exclusion }\end{array}$} & \multirow[t]{2}{*}{ TSD } \\
\hline & & & & & \\
\hline Norvège & $-6,0 \%$ & $10,2 \%$ & $17,9 \%$ & $5,8 \%$ & $27,9 \%$ \\
\hline Suède & $-6,6 \%$ & $14,2 \%$ & $14,7 \%$ & $5,5 \%$ & $27,8 \%$ \\
\hline Pays-Bas & $-6,6 \%$ & $15,4 \%$ & $14,7 \%$ & $4,2 \%$ & $27,7 \%$ \\
\hline Danemark & $-6,6 \%$ & $12,5 \%$ & $11,4 \%$ & $7,8 \%$ & $25,1 \%$ \\
\hline$\underline{\text { Finlande }}$ & $-6,6 \%$ & $12,2 \%$ & $11,5 \%$ & $5,1 \%$ & $22,2 \%$ \\
\hline \multicolumn{6}{|c|}{ Pays intermédiaires } \\
\hline Autriche & $-7,5 \%$ & $15,1 \%$ & $10,3 \%$ & $4,1 \%$ & $22,0 \%$ \\
\hline France & $-7,2 \%$ & $13,0 \%$ & $9,4 \%$ & $4,0 \%$ & $19,3 \%$ \\
\hline Allemagne & $-9,0 \%$ & $13,5 \%$ & $10,1 \%$ & $4,0 \%$ & $18,5 \%$ \\
\hline Tchéquie & $-4,8 \%$ & $9,9 \%$ & $10,0 \%$ & $2,7 \%$ & $17,8 \%$ \\
\hline Belgique & $-8,4 \%$ & $13,1 \%$ & $8,5 \%$ & $2,8 \%$ & $16,1 \%$ \\
\hline Royaume-Uni & $-10,8 \%$ & $10,8 \%$ & $9,4 \%$ & $3,7 \%$ & $13,1 \%$ \\
\hline \multicolumn{6}{|l|}{ Pays du Sud } \\
\hline Portugal & $-12,0 \%$ & $11,1 \%$ & $10,0 \%$ & $2,0 \%$ & $11,1 \%$ \\
\hline Italie & $-11,4 \%$ & $12,7 \%$ & $7,0 \%$ & $1,0 \%$ & $9,3 \%$ \\
\hline Grèce & $-12,3 \%$ & $11,2 \%$ & $6,4 \%$ & $2,5 \%$ & $7,8 \%$ \\
\hline Espagne & $-11,4 \%$ & $9,2 \%$ & $6,4 \%$ & $0,7 \%$ & $4,9 \%$ \\
\hline \multicolumn{6}{|c|}{ Pays de l'Europe centrale } \\
\hline Pologne & $-10,2 \%$ & $9,9 \%$ & $5,6 \%$ & $0,9 \%$ & $6,1 \%$ \\
\hline$\underline{\text { Lettonie }}$ & $-9,6 \%$ & $8,5 \%$ & $4,3 \%$ & $1,8 \%$ & $5,1 \%$ \\
\hline Slovaquie & $-12,6 \%$ & $7,1 \%$ & $7,5 \%$ & $2,6 \%$ & $4,6 \%$ \\
\hline Estonie & $-10,8 \%$ & $6,5 \%$ & $6,1 \%$ & $2,3 \%$ & $4,0 \%$ \\
\hline Lituanie & $-10,2 \%$ & $6,2 \%$ & $5,2 \%$ & $1,6 \%$ & $2,9 \%$ \\
\hline
\end{tabular}

Eurostat 2006

4. Les résultats en matière de pauvreté traduisent pour partie les niveaux différents de ressources démarchandisées. Dans les pays du Nord, là où les garanties de revenus sont plus indépendantes du marché du travail et où les politiques sociales visent davantage à l'intégration des personnes en vertu des droits issus de leur citoyenneté, les populations dont les revenus sont inférieurs au seuil de pauvreté sont relativement moins importantes. De 
même, dans les quatre pays continentaux "intermédiaires", les secours publics sont ancrés dans des traditions plus solides. D'où des niveaux plus limités d'insécurité économique dans ces deux groupes de pays, de $6 \%$ à $7 \%$ des Pays-Bas à la Finlande, et de $7 \%$ à $8 \%$ de la France à l'Allemagne. En revanche, au Royaume-Uni et au Portugal, là où la sécurité dépend plus du marché du travail et où les prestations sociales se limitent plus à un simple filet de sécurité, les pénalisations correspondant à l'insécurité économique sont près de deux fois plus importantes, de $11 \%$ à $12 \%$. De même, elles sont notables aussi, de $11 \%$ à $13 \%$, dans les autres pays du Sud aux redistributions encore très limitées. Quant aux PECO, la réduction importante des aides publiques assurées par l'État, le développement du nombre des chômeurs cessant d'être pris en charge par les entreprises et le développement tardif du mince "filet de sécurité" des protections sociales impliquent là aussi des niveaux importants de l'insécurité économique, de $10 \%$ à $13 \%{ }^{17}$.

\section{Avantages et limites des classements selon la sécurité démarchandisée}

Nous constatons que le taux de sécurité démarchandisée présente trois premiers avantages lorsque nous comparons les analyses des systèmes sociaux effectuées selon le TSD avec celles opérant soit avec l'indice de démarchandisation de Esping-Andersen soit avec la composante de sécurité de l'indicateur de bien-être économique de Osberg et Sharpe : $1^{\circ}$ il permet des comparaisons entre les systèmes sociaux de pays différents, alors même que leur niveau de développement est très inégal ; $2^{\circ}$ il aide à analyser l'importance des contributions des diverses prestations sociales à la sécurité économique des ressortissants, à la fois les unes par rapport aux autres et au niveau global, en parts du revenu disponible de référence $; 3^{\circ}$ il permet de construire des simulations de diverses modulations des politiques économiques et sociales. Mais ces avantages pratiques doivent être compris en fonction des limites non négligeables du TSD.

De premières incertitudes des tableaux et graphiques correspondent aux inadéquations des données issues de Eurostat pour évaluer les revenus démarchandisés. En particulier, en raison du caractère global des huit fonctions de protection sociale de la classification SESPROS, certaines aides en espèces ou en nature associées aux fonctions «chômage », «survie » et «vieillesse » sont agrégées à la catégorie des revenus de remplacement et non à celle des aides. Par ailleurs, la classification de Eurostat agrège tous les revenus contribuant à la constitution des retraites, qu'ils soient issus de régime de répartition ou de fonds de capitalisation, ce qui introduit un certain flou dans les comparaisons des revenus démarchandisés entre pays. De plus, en raison du caractère global des fonctions «maladie » et « invalidité » dans la classification SESPROS, cette catégorie comprend aussi les revenus de remplacement correspondant aux pensions d'invalidité et aux indemnités journalières associées aux arrêts maladie. D'où une imprécision supplémentaire de nos décompositions.

Au-delà de ces problèmes dont l'impact final est à évaluer, d'autres limites correspondent aux différences structurelles dans la répartition de l'emploi qui sont associées aux choix sociétaux des différents pays : entre générations (activité plus ou moins tardive des jeunes, inactivité plus ou moins précoce des personnes âgées), entre femmes et hommes et entre temps partiel et temps plein. Ces trois critères, largement interdépendants, pèsent de manière considérable sur le niveau du revenu moyen dans un pays en raison des différences de

\footnotetext{
${ }^{17}$ Il est significatif de constater que le classement des pays établi par Miles Corak (2006) sur les taux de faible revenu chez les enfants au début des années 2000, à partir des données réunis pour l'OCDE par Mira d'Ercole et Förster (2005), rejoint la hiérarchie des pays selon le TSD : seules la Grèce, la Pologne et l'Espagne apparaissent nettement mieux classée du point de vue du revenu des enfants que du TSD.
} 
rémunérations entre femmes et hommes, jeunes et personnes âgées, temps plein ou partiel. Par exemple, les proportions plus importantes de femmes actives et d'actifs âgés au Portugal, au Royaume-Uni et dans les pays du Nord se traduisent par deux facteurs contribuant à un niveau de sécurité économique plus élevé de ces différents pays : un plus important taux global d'activité, lequel rend possible une plus importante masse de cotisations, et une rémunération moyenne plus faible des actifs, d'où un dénominateur plus faible du $\mathrm{TSD}^{18}$. Le recours plus important au travail à temps partiel aux Pays-Bas, dans les pays du Nord et au Royaume Uni va dans le même sens et contribue à augmenter l'évaluation de la sécurité économique de ces pays.

Par ailleurs, un phénomène structurel de forte ampleur correspond à la part du travail souterrain non déclaré dans les pays du Sud tels que l'Italie, l'Espagne, la Grèce et les PECO. Ce phénomène est non négligeable en France également, ce qui implique une incertitude importante en ce qui concerne le taux de la population ayant un emploi. Même si ce flou implique une sous-estimation variable du TSD qu'il est difficile d'évaluer, on peut supposer qu'elle tend davantage à sous-estimer la sécurité dans les pays du Sud que dans ceux du Nord.

Les taux inégaux de propriété du logement principal, plus importants dans le Sud de l'Europe qu'au Royaume Uni ou dans les pays du Nord, concernent davantage les personnes âgées que les jeunes ménages. Ces taux entraînent donc des sous-estimations plus importantes des revenus et des prestations des inactifs au Sud qu'au Nord, ce qui contribue à une minoration plus importante du TSD chez les premiers que chez les seconds. De plus, les insécurités économiques associées aux logements locatifs, provisoires ou aux sans logis devraient pouvoir être prises en compte dans des évaluations plus étendues de l'insécurité économique.

Plus fondamentalement, dans la définition $d u T S D$, les conventions établissant la nature du revenu de référence par rapport auquel sont évaluées à la fois les différentes ressources démarchandisées et l'insécurité économique ont un poids très important. Elles ont été en partie justifiées plus haut. Mais leur caractère arbitraire demeure et de nombreuses autres possibilités pourraient être testées de manière alternative : le revenu moyen de l'ensemble des ménages, ou la médiane du revenu, soit en se limitant à la composante activité professionnelle comme dans nos calculs, soit en considérant l'ensemble des revenus ${ }^{19}$. Les simulations présentées ci-après montrent le caractère déterminant d'un tel choix.

\section{Principaux résultats des simulations des TSD de 20 pays européens en 2002}

Les acteurs sociaux peuvent se demander quelle seraient les conséquences sur la sécurité des populations d'une augmentation des allocations retraites ou des allocations chômage, ou encore des dépenses de remboursement des soins de santé. Ils peuvent aussi chercher à comparer les résultats de ces dépenses sur la sécurité avec ceux des augmentations de revenus d'un même montant qu'ils consentiraient aux salariés. C'est pour répondre à ces questions que nous avons réalisé une dizaine de simulations des déterminants du TSD.

\footnotetext{
${ }^{18}$ De ce point de vue, il serait intéressant d'évaluer des niveaux de revenu moyen à structures d'âge, de genre et de temps de travail comparables, ce qui permettrait de mesurer la contribution de ces choix sociétaux au classement relatif des niveaux de sécurité démarchandisée. De même, les évaluations du niveau du produit national par habitant en seraient sensiblement décalées. En raison de la constance de l'évaluation du produit en volume, ceci signifie simplement en effet que le niveau des prix relatifs serait décalé par une telle opération, traduisant ainsi la liaison des choix sociétaux à la fois avec la productivité du travail et avec les modalités de la répartition des revenus dans chaque pays.

${ }^{19}$ La place manque dans le présent article pour faire état des différents tests susceptibles de remédier à ces insuffisances. Ils pourraient éventuellement être intégrés dans une prochaine publication.
} 


\section{La méthode utilisée}

Afin d'évaluer la sensibilité du TSD aux variations des différentes prestations sociales ou des revenus des particuliers, nous avons simulé divers scénarios alternatifs de politique sociale. Nous avons retenu comme situation de référence l'état des 20 pays européens en 2002, tel qu'il s'est traduit dans les chiffres Eurostat. Faire varier de $1 \%$ un seul paramètre parmi les dix retenus vis-à-vis de leur situation en 2002 permet de calculer les élasticités ${ }^{20}$ de la sécurité économique par rapport aux variations des dix facteurs (sept diverses prestations sociales et trois types de revenus). Il faut rappeler à ce propos que les revenus salariaux, les revenus des indépendants et les revenus de la propriété sont les trois composantes principales du revenu disponible des actifs ayant un emploi, référence qui forme le dénominateur par rapport auquel sont estimées les ressources démarchandisées ${ }^{21}$.

Les variations des niveaux des élasticités de la sécurité correspondent à deux effets distincts : d'une part, l'effet direct des suppléments de prestations qui se traduit dans l'augmentation du niveau du numérateur; d'autre part, l'effet indirect impliqué par la modification du niveau de référence par rapport auquel la sécurité est évaluée, ce qui se traduit dans la modification à la fois du dénominateur et du niveau d'évaluation de l'insécurité économique des personnes vivant en dessous du seuil de pauvreté. La comparaison de ces deux effets nous aidera à interpréter les conséquences sur le TSD des simulations de politiques sociales.

\section{L'analyse des résultats des simulations}

Deux résultats généraux se dégagent de l'analyse des résultats du tableau 2. Dans celui-ci, dix colonnes donnent les élasticités du taux de sécurité démarchandisée (TSD) calculées pour des variations de $1 \%$ de chacune des sept principales prestations sociales et des trois principaux revenus directs, chaque facteur étant supposé varier seul sans modifier le niveau d'emploi. Les vingt pays européens sont ordonnés selon le niveau décroissant de leur TSD (dont le niveau en 2002 est rappelé dans la première colonne à gauche).

$1^{\circ}$ Les gains croissants de sécurité quand le niveau du TSD se réduit. D'une manière générale, les gains en matière de sécurité qui sont évalués par les élasticités apparaissent comme d'autant plus notables que le TSD initial est d'un niveau plus faible. Ce phénomène est essentiel si nous avons en tête les objectifs de cohésion sociale recherchés dans la stratégie de Lisbonne. Nous pouvons le vérifier pour les prestations démarchandisées ne touchant que les

\footnotetext{
${ }^{20}$ L'élasticité qualifie la sensibilité d'un objet donné par rapport à une force ou à une pression. En économie, on parle de l'élasticité de la demande d'un bien ou d'un service dépendant de divers facteurs (leurs niveaux de revenu, les prix, etc.). On calcule l'élasticité en effectuant le rapport des variations relatives de la variable considérée par rapport à celle du facteur que l'on veut étudier. Dans le cas de l'élasticité de la sécurité économique par rapport au montant des retraites, on a Élasticité de la sécurité économique = variation relative de la sécurité économique / variation relative du montant des retraites.

Par exemple, toutes choses égales par ailleurs, quand une augmentation de $1 \%$ des salaires en Pologne entraîne une baisse de 1,2\% de la sécurité, l'élasticité de la sécurité par rapport aux revenus est de -1,2. Dans ce cas-là, la baisse de la sécurité en pourcentage est plus importante que la hausse des revenus en pourcentage, et la sécurité est dite «élastique aux revenus ». En revanche, quand une augmentation de $1 \%$ des prestations famille entraîne une hausse en pourcentage moins élevée de la sécurité $(+0,12 \%)$, l'élasticité de la sécurité par rapport aux prestations familles $(0,12)$ est inférieure à l'unité ; on la dit alors « inélastique » à cette prestation.

21 Afin de prendre en compte les variations de sécurité impliquées par les charges fiscales associées aux suppléments des revenus imposables, on a endogénéisé le montant global des impôts à payer en considérant le taux de fiscalité comme constant et égal à celui de la situation de référence du pays considéré en 2002.
} 
personnes sans emploi (dont l'influence ne passe que par la variation du numérateur), pour les prestations versées sur une base universelle aux actifs et inactifs, et pour les différents revenus marchandisés (dont l'influence passe essentiellement par la variation du dénominateur).

$2^{\circ}$ Trois logiques distinctes gouvernent les variations des élasticités. Dans le tableau 2, les colonnes sont rangées de la gauche vers la droite selon un ordre d'indépendance décroissante à l'égard des marchés, en partant des retraites et du chômage, les prestations les plus indépendantes des revenus du travail, pour terminer par les revenus de l'activité professionnelle et de la propriété. C'est cet ordre que nous allons suivre pour analyser les rôles respectifs des facteurs directs et indirects dans les variations de la sécurité économique, ce qui nous permettra de constater qu'ils obéissent à trois logiques différentes.

\section{$\underline{1-\text { Les prestations concernant seulement les personnes sans emploi }}$}

Les augmentations de ces prestations qui se concentrent sur les inactifs (chômage et vieillesse) ont l'effet sécurisant le plus important, a fortiori lorsque ces prestations se développent dans un pays déjà caractérisé par un faible niveau général de la couverture sociale (pays du Sud et PECO, voir 9 dernières lignes du tableau 2). Les prestations chômage ou vieillesse constituent en effet des prestations démarchandisées par excellence dans la mesure où elles ne bénéficient qu'aux personnes retirées du marché du travail et n'affectent pas directement le revenu des personnes ayant un emploi. Les variations de leur contribution au TSD passent donc uniquement par l'augmentation des revenus entrant dans le numérateur.

Les prestations associées à la lutte contre l'exclusion (colonne 3) contribuent de la même manière au TSD car elles ne concernent que les inactifs; elles ne jouent donc que sur le numérateur du TSD. Là encore, elles jouent un rôle plus important en Slovaquie et en Lituanie, pays où ces prestations sont encore très peu développées même si elles y sont d'un montant moins important que les retraites ou les allocations chômage. Il faut noter aussi que le rôle sécurisant d'une même augmentation des prestations est pondéré par le niveau de vie : plus il sera d'un faible niveau, plus un même accroissement du RMI par exemple se traduira dans une augmentation plus importante de la sécurité, et donc par une forte élasticité.

Afin d'illustrer ces calculs, nous avons imaginé des exemples plausibles dans les conditions sociales actuelles, ceux d'une croissance de $2 \%$ des allocations retraite ou de $2 \%$ des allocations chômage. En Pologne, par exemple, un accroissement de $2 \%$ des retraites en 2002 entraînerait une augmentation du TSD de 2,7 \%. Alors qu'au Danemark, dont le niveau de vie et les retraites sont bien supérieures, cette même augmentation ne serait liée qu'à une hausse de $0,8 \%$ de la sécurité démarchandisée. Pour une augmentation de $2 \%$ des dépenses publiques liées au chômage, déjà considérables au Danemark (un quart des retraites), le progrès du TSD ne serait que de $0,18 \%$ (un quart du taux également). En revanche, en Pologne, les progrès de la sécurité démarchandisée associés à ce même accroissement ne seraient respectivement que de $0,23 \%$, car les allocations chômages représentent une part bien plus limitée des prestations sociales dans ce pays. 
Tableau 2 : Élasticités du TSD par rapport aux variations de prestations sociales et de revenus primaires

\begin{tabular}{|c|c|c|c|c|c|c|c|c|c|c|c|}
\hline \multirow[b]{2}{*}{ Pays } & \multirow[b]{2}{*}{$\begin{array}{l}\text { TSD net } \\
\text { en } 2002\end{array}$} & \multicolumn{3}{|c|}{ Prestation versée aux sans emploi slmt } & \multicolumn{4}{|c|}{ Prestation versée aux actifs et inactifs } & \multicolumn{3}{|c|}{ Supplément de revenu } \\
\hline & & $\begin{array}{c}1 \\
1 \\
\text { Suppl. Prest. } \\
\text { Retraite av. } \\
\text { Suppl. impôt }\end{array}$ & $\begin{array}{c}2 \\
\text { Suppl. Prest. } \\
\text { Chômage av. } \\
\text { Suppl. impôt }\end{array}$ & $\begin{array}{c}3 \\
\text { Suppl. Prest. } \\
\text { exclusion, } \\
\text { sans Suppl. } \\
\text { impôt }\end{array}$ & \begin{tabular}{|c|} 
\\
Suppl. Prest. \\
famille, sans \\
Suppl. impôt
\end{tabular} & $\begin{array}{c}5 \\
5 \\
\text { Suppl. Prest. } \\
\text { logement, } \\
\text { sans Suppl. } \\
\text { impôt }\end{array}$ & $\begin{array}{c}6 \\
\text { Suppl. Prest. } \\
\text { maladie av. } \\
\text { Suppl. impôt }\end{array}$ & $\begin{array}{c}7 \\
\text { Suppl. Prest. } \\
\text { invalidité av. } \\
\text { Suppl. impôt }\end{array}$ & $\begin{array}{c}8 \\
\text { Suppl. de } \\
\text { salaire à } \\
\text { emploi fixe }\end{array}$ & \begin{tabular}{|c|}
9 \\
\\
Suppl. de \\
Rev. indépdt \\
à emploi fixe
\end{tabular} & \begin{tabular}{|c|}
10 \\
\\
Suppl. de \\
Revenus de \\
la propriété
\end{tabular} \\
\hline Norvège & $27,9 \%$ & 0,32 & 0,03 & 0,03 & 0,11 & 0,01 & 0,28 & 0,14 & $-0,70$ & $-0,14$ & $-0,09$ \\
\hline Suède & $27,8 \%$ & 0,42 & 0,07 & 0,03 & 0,09 & 0,02 & 0,23 & 0,12 & $-0,77$ & $-0,18$ & $-0,04$ \\
\hline Pays-Bas & $27,7 \%$ & 0,43 & 0,06 & 0,07 & 0,04 & 0,01 & 0,26 & 0,09 & $-0,66$ & $-0,19$ & $-0,18$ \\
\hline Danemark & $25,1 \%$ & 0,40 & 0,10 & 0,06 & 0,15 & 0,03 & 0,19 & 0,12 & $-0,82$ & $-0,13$ & $-0,08$ \\
\hline Finlande & $22,2 \%$ & 0,39 & 0,12 & 0,03 & 0,13 & 0,01 & 0,24 & 0,13 & $-0,84$ & $-0,18$ & $-0,07$ \\
\hline Autriche & $22,0 \%$ & 0,60 & 0,07 & 0,02 & 0,11 & 0,00 & 0,25 & 0,08 & $-0,77$ & $-0,26$ & $-0,11$ \\
\hline France & $19,3 \%$ & 0,49 & 0,10 & 0,02 & 0,10 & 0,03 & 0,31 & 0,05 & $-0,79$ & $-0,31$ & $-0,10$ \\
\hline Allemagne & $18,5 \%$ & 0,58 & 0,12 & 0,03 & 0,13 & 0,01 & 0,31 & 0,09 & $-0,83$ & $-0,24$ & $-0,22$ \\
\hline Tchéquie & $17,8 \%$ & 0,50 & 0,04 & 0,04 & 0,08 & 0,01 & 0,35 & 0,08 & $-0,72$ & $-0,35$ & $-0,05$ \\
\hline Belgique & $16,1 \%$ & 0,49 & 0,17 & 0,03 & 0,11 & 0,00 & 0,29 & 0,11 & $-0,91$ & $-0,29$ & $-0,15$ \\
\hline Royaume-Uni & $13,1 \%$ & 0,72 & 0,05 & 0,02 & 0,11 & 0,09 & 0,41 & 0,14 & $-1,16$ & $-0,24$ & $-0,18$ \\
\hline Portugal & $11,1 \%$ & 0,78 & 0,08 & 0,04 & 0,11 & 0,00 & 0,50 & 0,19 & $-1,28$ & $-0,41$ & $-0,14$ \\
\hline Italie & $9,3 \%$ & 1,11 & 0,04 & 0,00 & 0,08 & 0,00 & 0,48 & 0,11 & $-1,04$ & $-0,69$ & $-0,31$ \\
\hline Grèce & $7,8 \%$ & 1,18 & 0,16 & 0,06 & 0,15 & 0,05 & 0,55 & 0,11 & $-0,78$ & $-1,47$ & $-0,09$ \\
\hline Espagne & $4,9 \%$ & 1,36 & 0,43 & 0,03 & 0,08 & 0,02 & 0,88 & 0,22 & $-2,00$ & $-0,96$ & $-0,14$ \\
\hline Pologne & $6,1 \%$ & 1,37 & 0,11 & 0,01 & 0,12 & 0,00 & 0,47 & 0,29 & $-1,19$ & $-1,15$ & $-0,14$ \\
\hline Slovaquie & $4,6 \%$ & 1,37 & 0,15 & 0,23 & 0,26 & 0,01 & 1,07 & 0,28 & $-1,93$ & $-1,34$ & $-0,13$ \\
\hline Lettonie & $5,1 \%$ & 1,51 & 0,09 & 0,02 & 0,27 & 0,02 & 0,50 & 0,24 & $-1,85$ & $-0,47$ & $-0,39$ \\
\hline Estonie & $4,0 \%$ & 1,53 & 0,04 & 0,08 & 0,39 & 0,02 & 0,99 & 0,28 & $-2,65$ & $-0,58$ & $-0,12$ \\
\hline Lituanie & $2,9 \%$ & 1,98 & 0,08 & 0,17 & 0,35 & 0,00 & 1,20 & 0,37 & $-2,48$ & $-1,06$ & $-0,68$ \\
\hline
\end{tabular}

Lecture: en 2002, une augmentation relative du montant des prestations retraite de $1 \%$ entraîne une augmentation relative du TSD de 0,32\% en Norvège alors qu'elle implique une augmentation relative de 1,37\% du TSD en Pologne. En revanche, une variation relative des salaires de $1 \%$ entraîne une diminution relative du TSD de 2,48\% en Lituanie, alors qu'elle n'implique qu'une diminution relative de 0,7\% en Norvège.

\section{2 - Les prestations affectées indépendamment de la situation par rapport à l'emploi}

Les prestations maladie et invalidité (colonnes 6 et 7) sont à vocation universelle et concernent à la fois les personnes en emploi et celles qui sont en dehors du marché du travail. Ces prestations ont un effet sécurisant (TSD) très significatif là où elles sont quasi inexistantes (dans les pays du Sud et les PECO). Il faut noter toutefois que leur effet sécurisant est moins important que celui des prestations concentrées sur les personnes en dehors de l'emploi. Leur influence est en effet diminuée par le fait qu'elles contribuent à augmenter le revenu de référence des actifs, dont le niveau joue à la fois sur le dénominateur du TSD et sur l'évaluation de l'insécurité économique.

Les aides à la famille et au logement (colonnes 4 et 5) concernent également à la fois les personnes en emploi et celles qui sont en dehors du marché du travail mais elles sont fréquemment attribuées sur des conditions de ressources. Leur augmentation a donc un effet sur le TSD plus limité car l'accroissement de leur contribution au numérateur est plus faible et de plus compensé en partie par le fait qu'elles s'ajoutent aux revenus des actifs, ce qui augmente le revenu de référence et par conséquent aussi le dénominateur. Dans la mesure où ces prestations sont très limitées actuellement dans les pays du Sud et les PECO, leur contribution au TSD de ces derniers est plus importante.

\section{3 - Les revenus de l'activité et de la propriété}

Les suppléments de rémunérations à effectif constant des personnes employées et les progrès du niveau de vie des actifs se traduisent en revanche dans une diminution de la sécurité démarchandisée (TSD). Pourquoi ? Parce que ces augmentations entrainent celle du revenu de référence, lequel permet d'évaluer à la fois l'insécurité économique et la sécurité 
démarchandisée procurée par les prestations. Quand le niveau de vie augmente, la sécurité démarchandisée procurée par un certain montant de prestations relativement au revenu de référence diminue. Le tableau 2 indique que ces diminutions sont d'autant plus importantes que les dénominateurs considérés sont plus faibles : plus pour les pays de l'Est ou du Sud que pour les pays nordiques. Un deuxième effet considérable joue au numérateur : il s'agit de l'augmentation de l'insécurité économique qui pénalise d'autant plus la sécurité démarchandisée que le taux de pauvreté et le revenu de référence sont plus importants.

Ces deux facteurs expliquent le caractère négatif des élasticités correspondant aux revenus de l'activité et de la propriété (3 colonnes de droite du tableau 2). Les revenus de la propriété (colonne 10) sont partagés entre les actifs et les inactifs, et ils affectent donc moins le revenu de référence ; ceci explique que les élasticités correspondantes soient plus faibles que celles des revenus indépendants, pourtant d'une importance comparable.

Les revenus salariaux (colonne 8) constituent la plus importante part du revenu de référence, ce qui explique que les élasticités associées sont toujours les plus importantes. Une seule exception, la Grèce, pays où la part du travail informel est très importante et où les revenus du travail indépendant comptabilisés sont supérieurs à ceux du travail salarié. La bien plus faible importance des élasticités des pays du Nord par rapport à celles des PECO, des pays du Sud ou du Royaume-Uni s'explique à la fois par le niveau plus élevé des revenus dans ces pays, ce qui élève d'autant la référence de niveau de vie, et par leur plus faible taux de pauvreté. Comparé au niveau de la Norvège $(-0,70)$, la plus forte pénalisation du Royaume-Uni $(-1,16)$ s'explique largement par ce deuxième aspect, alors que celles de l'Espagne $(-2,00)$ ou de l'Estonie $(-2,65)$ combinent les deux.

\section{Trois logiques déterminent la sensibilité de la sécurité aux variations de ses composantes}

Trois logiques différentes apparaissent quand nous comparons les sensibilités de la sécurité démarchandisée aux différentes prestations et sources de revenus qui la déterminent. Les caractères des trois logiques d'actions de ces composantes sont illustrés par le tableau 3 qui réalise une synthèse entre de multiples informations ${ }^{22}$. Il est remarquable d'y constater que le rapport des élasticités ne prend que trois valeurs pour chacun des pays : soit un rendement maximum de 1 , pour les trois prestations qui ne concernent que les personnes en dehors de l'emploi (colonne 1, 2, 3) et qui, n'influant pas sur le revenu des actifs, ne voient pas leurs effets directs atténués ; soit un rendement inférieur à 1 pour les quatre prestations à vocation universelle (col. 4 à 7) dont l'effet sur la sécurité est atténué par l'augmentation associée du niveau du revenu de référence, à la fois à travers l'insécurité et par le dénominateur ; soit un coefficient supérieur à 1 pour les trois revenus n'entrant pas dans la sécurité démarchandisée (col. 8, 9, 10) mais dont l'augmentation contribue néanmoins à démultiplier la minoration associée à l'insécurité économique (proportionnelle au revenu des actifs) du fait de la division par le dénominateur, toutes choses qui contribuent à la diminution du TSD ${ }^{23}$.

\footnotetext{
${ }^{22}$ Pour évaluer le rendement des prestations et la sensibilité aux revenus, nous calculons simplement les rapports de l'élasticité du TSD et de l'élasticité du numérateur du TSD, ceci pour les dix composantes des ressources de sécurité. Une telle opération revient à mettre en rapport les effets globaux des prestations sur la sécurité démarchandisée (effets à la fois directs et indirects sur le revenu de référence par rapport auquel la sécurité et l'insécurité sont évaluées) avec le seul effet sur le numérateur. Les étapes de ces calculs concernent trois termes: les élasticités de la sécurité démarchandisée (tableau 2) et les élasticités des numérateurs et dénominateurs du TSD (reproduits dans les tableaux 4 et 5 de l'annexe 2).

${ }^{23}$ Un tel coefficient ne peut être interprété en terme de rendement puisqu'il évalue l'effet indirect de l'augmentation des revenus sur la sécurité démarchandisée, revenus qui ne sont pas des sources de la sécurité.
} 
Tableau 3 : Deux types de rendement des prestations en matière de sécurité

\begin{tabular}{|c|c|c|c|c|c|c|c|c|c|c|c|}
\hline \multirow[b]{2}{*}{ Pays } & \multirow[b]{2}{*}{$\begin{array}{c}\text { TSD net en } \\
2002\end{array}$} & \multicolumn{3}{|c|}{ Prestation versée aux sans emploi slmt } & \multicolumn{4}{|c|}{ Prestation versée aux actifs et inactifs } & \multicolumn{3}{|c|}{ Supplément de revenu } \\
\hline & & $\begin{array}{c}1 \\
\text { Suppl. Prest. } \\
\text { Retraite av. } \\
\text { Suppl. impôt }\end{array}$ & $\begin{array}{c} \\
\\
\text { Suppl. Prest. } \\
\text { Chômage av. } \\
\text { Suppl. impôt }\end{array}$ & $\begin{array}{c}3 \\
\text { Suppl. Prest. } \\
\text { exclusion, } \\
\text { sans Suppl. } \\
\text { impôt }\end{array}$ & $\begin{array}{c} \\
\\
\text { Suppl. Prest. } \\
\text { famille, sans } \\
\text { Suppl. impôt }\end{array}$ & $\begin{array}{c}5 \\
\text { Suppl. Prest. } \\
\text { logement, } \\
\text { sans Suppl. } \\
\text { impôt }\end{array}$ & $\begin{array}{c}6 \\
\text { Suppl. Prest. } \\
\text { maladie av. } \\
\text { Suppl. impôt }\end{array}$ & $\begin{array}{c}7 \\
\text { Suppl. Prest. } \\
\text { invalidité av. } \\
\text { Suppl. impôt }\end{array}$ & $\begin{array}{c} \\
\\
\text { Suppl. de } \\
\text { salaire à } \\
\text { emploi fixe }\end{array}$ & \begin{tabular}{|c|}
9 \\
Suppl. de Rev. \\
indépdt à \\
emploi fixe
\end{tabular} & \begin{tabular}{|c|}
10 \\
Suppl. de \\
Revenus de la \\
propriété
\end{tabular} \\
\hline Norvège & $27,9 \%$ & 1,00 & 1,00 & 1,00 & 0,70 & 0,70 & 0,70 & 0,70 & 5,61 & 5,64 & 5,64 \\
\hline Suède & $27,8 \%$ & 1,00 & 1,00 & 1,00 & 0,70 & 0,70 & 0,70 & 0,70 & 5,18 & 5,20 & 5,21 \\
\hline Pays-Bas & $27,7 \%$ & 1,00 & 1,00 & 1,00 & 0,70 & 0,70 & 0,70 & 0,70 & 5,17 & 5,19 & 5,19 \\
\hline Danemark & $25,1 \%$ & 1,00 & 1,00 & 1,00 & 0,73 & 0,73 & 0,73 & 0,73 & 4,77 & 4,80 & 4,80 \\
\hline Finlande & $22,2 \%$ & 1,00 & 1,00 & 1,00 & 0,76 & 0,76 & 0,76 & 0,76 & 4,34 & 4,36 & 4,36 \\
\hline Autriche & $22,0 \%$ & 1,00 & 1,00 & 1,00 & 0,76 & 0,76 & 0,76 & 0,76 & 3,92 & 3,93 & 3,93 \\
\hline France & $19,3 \%$ & 1,00 & 1,00 & 1,00 & 0,79 & 0,79 & 0,79 & 0,79 & 3,66 & 3,67 & 3,67 \\
\hline Allemagne & $18,5 \%$ & 1,00 & 1,00 & 1,00 & 0,80 & 0,80 & 0,80 & 0,80 & 3,04 & 3,05 & 3,05 \\
\hline Tchéquie & $17,8 \%$ & 1,00 & 1,00 & 1,00 & 0,81 & 0,81 & 0,81 & 0,81 & 4,68 & 4,70 & 4,71 \\
\hline Belgique & $16,1 \%$ & 1,00 & 1,00 & 1,00 & 0,82 & - & 0,82 & 0,82 & 2,90 & 2,91 & 2,91 \\
\hline Royaume-Uni & $13,1 \%$ & 1,00 & 1,00 & 1,00 & 0,85 & 0,85 & 0,85 & 0,85 & 2,20 & 2,21 & 2,21 \\
\hline Portugal & $11,1 \%$ & 1,00 & 1,00 & 1,00 & 0,87 & 0,87 & 0,87 & 0,87 & 1,91 & 1,92 & 1,92 \\
\hline Italie & $9,3 \%$ & 1,00 & 1,00 & 1,00 & 0,89 & 0,90 & 0,89 & 0,89 & 1,81 & 1,81 & 1,81 \\
\hline Grèce & $7,8 \%$ & 1,00 & 1,00 & 1,00 & 0,91 & 0,91 & 0,91 & 0,91 & 1,63 & 1,63 & 1,64 \\
\hline Espagne & $4,9 \%$ & 1,00 & 1,00 & 1,00 & 0,95 & 0,95 & 0,94 & 0,95 & 1,42 & 1,42 & 1,43 \\
\hline Pologne & $6,1 \%$ & 1,00 & 1,00 & 1,00 & 0,93 & - & 0,93 & 0,93 & 1,59 & 1,59 & 1,60 \\
\hline Slovaquie & $4,6 \%$ & 1,00 & 1,00 & 1,00 & 0,95 & 0,95 & 0,95 & 0,95 & 1,36 & 1,36 & 1,36 \\
\hline Lettonie & $5,1 \%$ & 1,00 & 1,00 & 1,00 & 0,94 & 0,94 & 0,94 & 0,94 & 1,52 & 1,52 & 1,52 \\
\hline Estonie & $4,0 \%$ & 1,00 & 1,00 & 1,00 & 0,95 & 0,95 & 0,95 & 0,95 & 1,36 & 1,37 & 1,37 \\
\hline Lituanie & $2,9 \%$ & 1,00 & 1,00 & 1,00 & 0,97 & 0,97 & 0,97 & 0,97 & 1,28 & 1,28 & 1,28 \\
\hline
\end{tabular}

Eurostat 2006

Lecture : dans tous les pays, une variation des prestations de retraite (col. 1) n'entraîne qu'une variation du numérateur du TSD, le dénominateur restant constant. L'élasticité du TSD pour ces prestations est donc égale à l'élasticité du numérateur et le rendement de ces prestations, que mesure le rapport des élasticités, est égal à 1 . De même pour les prestations chômage (col. 2) ou relative à l'exclusion sociale (col. 3). En revanche, une variation des prestations famille (col. 4) contribue à la fois à augmenter le revenu des inactifs et celui des actifs, d'ou une augmentation du TSD plus faible du fait de la croissance du dénominateur, le rendement final de ces prestations n'étant que de 0,7 dans le cas de la Norvège en 2002. Enfin, un supplément de revenu à prestations constantes (col. 8) implique à la fois une diminution du numérateur du fait de l'augmentation de l'insécurité économique proportionnelle au revenu des actifs en emploi et une augmentation du dénominateur qui contribue également à diminuer la sécurité démarchandisée ; en Lituanie, l'accroissement de l'un ou l'autre des revenus a un rendement supérieur à 1 puisqu'il entraîne ainsi une diminution de l'élasticité du TSD 1,28 fois plus importante que la diminution relative du numérateur.

Les différences entre les rendements des différentes prestations en matière de sécurité démarchandisée suggèrent en quoi les politiques ciblant directement les personnes sans emploi ont un intérêt plus important pour la sécurité des populations. Ce constat est plus valable pour les pays du Nord et pour les pays intermédiaires comme nous pouvons le constater en notant dans le tableau 3 la croissance du rendement des prestations à vocation universelle au fur et à mesure que le TSD décroît. A la limite, dans les PECO, le rendement supérieur à $93 \%$ de toutes les prestations montre que toutes les politiques sociales contribuent d'une manière à peu près semblable à augmenter la sécurité des populations et à augmenter la cohésion sociale, ce qui va dans le sens des recommandations de la stratégie de Lisbonne.

Au-delà des différences entre pays, l'opposition entre ces trois logiques met en évidence l'importance qu'il faut accorder aux évolutions des revenus de référence par rapport auxquels les populations apprécient ou déprécient les prestations dont elles bénéficient. Les gains de sécurité procurés par un même montant de prestations sont d'autant plus importants qu'ils concernent des mesures associées avec une hausse plus limitée du niveau général des revenus. 


\section{CONCLUSION}

En définitive, la revue des modes de prise en compte de la sécurité démarchandisée dans les pays européens nous a montré que cette dimension apparaît comme un apport fondamental des États sociaux et qu'il est utile d'en distinguer les contributions selon quatre groupes de pays. La sécurité démarchandisée est spécialement développée dans les États sociauxdémocrates du Nord, là où les conquêtes sociales se sont le plus institutionnalisées de façon largement indépendante des contraintes du marché du travail. Ce caractère légitime d'autant plus l'appellation "démarchandisée" donnée à ces formes de sécurité. Autre résultat remarquable: les politiques sociales plus fondées sur la sécurité démarchandisée au Nord aboutissent à davantage d'intégration des personnes du fait des droits issus de leur citoyenneté, ce qui se traduit dans des taux de pauvreté pratiquement inférieurs de moitié. En revanche, au Royaume-Uni et au Portugal, là où la sécurité est plus "marchandisée", dans le sens où elle dépend plus du marché du travail, les proportions de pauvres sont deux fois plus importantes que dans les pays sociaux-démocrates. Néanmoins, tout se passe comme si un patrimoine social perdurait dans le vieil État social du Royaume Uni puisque l'importance des revenus sociaux hérités du plan Beveridge y maintient le taux de sécurité démarchandisée largement au-dessus de celui de l'Italie. Nous avons constaté enfin le bas niveau des sécurités démarchandisées dans les pays d'Europe continentale et orientale. Raison de plus pour s'attacher à prendre en compte la dimension de la sécurité dans la définition des systèmes de protection sociale que ces nations s'apprêtent à développer.

Il peut être utile pour cela de s'appuyer sur nos simulations des politiques sociales. Elles nous ont incité à distinguer deux types de prestations: celles qui sont dirigées sur les personnes sans emploi et les prestations à vocation universelle dont les effets moins ciblés contribuent moins à accroître le niveau des sécurités démarchandisées. Ces deux types de prestations aident plus à la convergence des systèmes sociaux quand elles sont distribuées dans les pays du Sud et les PECO dont les systèmes sociaux sont significativement moins développés que dans les États sociaux du Nord de l'Europe. Le rendement plus important des prestations dirigées vers les populations sans emploi, particulièrement celles attribuées à la lutte contre l'exclusion, suggère ainsi quelles politiques seraient les plus à même de renforcer la cohésion sociale, ce qui est recherché par la stratégie de Lisbonne.

Nos simulations nous ont aussi permis de mettre en évidence l'importance des liens entre les politiques de redistribution et les effets indirects qu'elles génèrent à travers la détermination des revenus de référence sur lesquels les populations s'appuient pour apprécier leur niveau de vie. Elles ont montré aussi les relations complexes qui associent les taux de pauvreté, l'appréciation des niveaux d'insécurité associés et la répartition de la population entre salariés, indépendants et sans emploi. L'approfondissement de ces relations peut devenir de plus en plus d'actualité avec le développement de la mondialisation des échanges et des modes de production.

\section{BIBLIOGRAPHIE}

Castel R. (2003), L'insécurité sociale. Qu'est-ce qu'être protégé ?, Seuil, Paris.

Corak M. (2006), "Principes et aspects pratiques de la mesure de la pauvreté chez les enfants", dans Revue internationale de sécurité sociale, vol. $59, \mathrm{n}^{\circ} 2$.

Esping-Andersen G. (1990), The Three Worlds of Welfare Capitalism, Cambridge, Polity Press, Trad. : Les trois mondes de l'État-providence, Paris, PUF, 1999.

Euzéby C. (2004), "Repenser la protection sociale dans l'Union européenne : vers plus de droits fondamentaux", dans Revue internationale de sécurité sociale, vol. 57, n 1 .

Gadrey J. ; Jany-Catrice F. (2003), « Développement et progrès social, quels indicateurs 
choisir ? », dans Alternatives économiques, $\mathrm{n}^{\circ}$ 211, février, pp. 70-73

Hobbes T. (1651), Léviathan - La matière, la forme et le pouvoir d'une république ecclésiastique et civile, Traduit de l'anglais par Philippe Folliot, à partir de Leviathan or the Matter, Forme and Power of A Commonwealth Ecclesiastical and civil, London, Green Dragon, St. Pauls Church-yard.

Lewis J. (1992), «Gender and the Development of Welfare Regimes », Journal of European Social Policy, 3, pp. 159-173.

Locke J. (1669), Second traité de gouvernement, Trad. Fr., 1994, PUF, Paris.

Menahem G. (2006), "Deux indicateurs du développement des droits sociaux : indicateur de démarchandisation versus taux de sécurité économique ", dans AES, Économie sociale et droit, XXVIe journées de l'Association d'Économie Sociale, 7 et 8 septembre, Éditions l'Harmattan, pp. 321-331.

Menahem G. ; Cherilova V. (2005), "Inégalités de sécurité économique et aide à la famille dans l'Union européenne", dans Recherches et prévisions, $\mathrm{n}^{\circ} 79$, pp. 83-95.

Merrien F.-X. (2005), L'État social, Armand-Colin, Paris.

Mira d'Ercole M. ; Förster M. (2005), Income distribution and poverty in OECD countries in the second half of the 1990s. Documents de travail de l'OCDE n²2. Paris.

Orloff A. S. (1993), "Gender and the Social Rights of Citizenship: the Comparative Analysis of Gender Relations and Welfare States", American Sociological Review, 58, USA, pp. 303-328.

Osberg L. ; Sharpe A. (2000), "An Index of Economic Well-being for Selected OECD Countries", Review of Income and Wealth, Groningen, Netherlands.

Rhodes M.; Palier B. (1997), "Conclusion générale", dans "Rencontres de Florence Comparer les systèmes de protection sociale en Europe du Sud", Revue française des affaires sociales, $57^{\text {ème }}$ année, 4(numéro spécial).

Standing G. (2002), "Enquêtes sur la sécurité des personnes: vers un indicateur du travail décent", Revue internationale du travail, volume 141, 4.

Strobel P. (2003), "Présentation : le modèle nordique de protection sociale sous le choc des réformes", dans "L'État providence nordique", Revue française des affaires sociales, $57^{\text {ème }}$ année, 4(numéro spécial).

Polanyi K. (1944), The Great Transformation, Beacon Press, Boston, USA.

Remerciements : Je remercie les deux referees anonymes et le professeur Jean Gadrey pour le soin de leur relecture et la qualité des remarques qu'ils ont formulées, lesquelles m'ont incité à apporter de considérables améliorations à mon projet d'article initial. Je suis bien entendu seul responsable des erreurs et insuffisances qui pourraient subsister. 


\section{ANNEXES}

\section{Une source : Eurostat}

Eurostat et, en particulier, sa base NewCronos ont permis de rassembler des statistiques des populations, des différents revenus et prestations sociales et des taux de pauvreté pour treize pays de l'Union européenne. À la date des interrogations, on ne disposait de chiffres homogènes pour les différents revenus et prestations que pour les années 1990 à 2000. Les statistiques de revenu disponible n'étant pas accessibles pour le Luxembourg et l'Irlande, on a dû se limiter à treize des quinze pays de l'Union européenne en 2000. Ces statistiques n'ont pu être rassemblées pour les dix années que pour neuf pays, les données de l'Allemagne n'étant disponibles qu'à partir de 1991, celles de la Suède qu'à partir de 1993 et celles de l'Autriche, de l'Espagne, de la Grèce et du Portugal qu'à partir de 1995.

La disponibilité des statistiques de seuil de pauvreté est encore plus restreinte, ce qui a limité l'étendue des calculs du TSD. L'évolution du TSD est ainsi calculée sur six années pour onze pays (de 1995 à 2000), sur cinq années pour douze, et sur quatre années pour les treize pays.

On a utilisé la nomenclature SESPROS des prestations sociales en huit postes établie et harmonisée pour la base NewCronos. Quelles que soient les limites de cette nomenclature, elle a l'avantage de permettre de comparer des systèmes très différents, tant par la nature des droits sociaux qui les fondent que par le contenu des prestations qu'ils génèrent ou par l'orientation des politiques qu'ils soutiennent.

\section{Les étapes du calcul du TSD et du rendement des prestations}

Le TSD est un taux qui évalue un numérateur, la somme des ressources de sécurité démarchandisée moins l'insécurité économique, relativement à un dénominateur, le revenu disponible moyen des actifs en emploi. La connaissance des élasticités du numérateur (voir tableau 4) et du dénominateur (tableau 5) est donc doublement utile.

Elle permet d'abord de comprendre la logique de la construction de l'élasticité du TSD. Du fait que l'élasticité d'un rapport est égale à la différence entre l'élasticité du numérateur et celle du dénominateur, chaque cellule du tableau 2 se déduit logiquement de la différence entre les cellules analogues des tableaux 4 et 5 . En Norvège, par exemple, l'élasticité de la sécurité démarchandisée par rapport au montant des prestations maladie (col. 8) est, selon le tableau 2, de 0,28 . Quand nous constatons sur les tableaux 4 et 5 que ce chiffre est égal à la différence entre l'élasticité du numérateur $(0,40)$ et l'élasticité du dénominateur $(0,12)$, nous comprenons mieux les origines de sa différence avec l'élasticité de la Lituanie $(1,20$, soit respectivement la différence entre 1,24 et 0,04 ), laquelle est à mettre en rapport avec le très faible développement des prestations maladie dans ce pays. 
Tableaux 4 et 5 : Élasticités du numérateur et du dénominateur du TSD

Elasticités du numérateur du TSD = (somme des prestations sociales) - (Insécurité économique =[Nb pauvres] $]^{*}[$ Revenu de référence])

\begin{tabular}{|c|c|c|c|c|c|c|c|c|c|c|c|}
\hline \multirow[b]{2}{*}{ Pays } & \multirow[b]{2}{*}{$\begin{array}{c}\text { TSD en } \\
2002\end{array}$} & \multicolumn{3}{|c|}{ Prestation versée aux sans emploi slmt } & \multicolumn{4}{|c|}{ Prestation versée aux actifs et inactifs } & \multicolumn{3}{|c|}{ Supplément de revenu } \\
\hline & & $\begin{array}{c}1 \\
\text { Suppl. Prest. } \\
\text { Retraite av. } \\
\text { Suppl. impôt }\end{array}$ & $\begin{array}{c}2 \\
\text { Suppl. Prest. } \\
\text { Chômage av. } \\
\text { Suppl. impôt }\end{array}$ & $\begin{array}{c}3 \\
\text { Suppl. Prest. } \\
\text { exclusion , } \\
\text { sans Suppl. } \\
\text { impôt }\end{array}$ & $\begin{array}{c}4 \\
\text { Suppl. Prest. } \\
\text { famille, sans } \\
\text { Suppl. impôt }\end{array}$ & $\begin{array}{c}5 \\
\text { Suppl. Prest. } \\
\text { logement, } \\
\text { sans Suppl. } \\
\text { impôt }\end{array}$ & \begin{tabular}{|c}
6 \\
Suppl. Prest. \\
maladie av. \\
Suppl. impôt
\end{tabular} & $\begin{array}{c}7 \\
\text { Suppl. Prest. } \\
\text { invalidité av. } \\
\text { Suppl. impôt }\end{array}$ & $\begin{array}{c}8 \\
\text { Suppl. de } \\
\text { salaire à } \\
\text { emploi fixe }\end{array}$ & $\begin{array}{c}9 \\
\text { Suppl. de } \\
\text { Rev. indépdt } \\
\text { à emploi fixe }\end{array}$ & $\begin{array}{c}10 \\
\text { Suppl. de } \\
\text { Revenus de } \\
\text { la propriété }\end{array}$ \\
\hline Norvège & & 0,32 & 0,03 & 0,03 & 0,16 & 0,01 & 0,40 & 0,20 & $-0,12$ & $-0,03$ & $-0,02$ \\
\hline Suède & & 0,42 & 0,07 & 0,03 & 0,13 & 0,03 & 0,33 & 0,17 & $-0,15$ & $-0,04$ & $-0,01$ \\
\hline Pays-Bas & & 0,43 & 0,06 & 0,07 & 0,06 & 0,02 & 0,36 & 0,13 & $-0,13$ & $-0,04$ & $-0,03$ \\
\hline Danemark & & 0,40 & 0,10 & 0,06 & 0,20 & 0,03 & 0,26 & 0,16 & $-0,17$ & $-0,03$ & $-0,02$ \\
\hline Finlande & & 0,39 & 0,12 & 0,03 & 0,17 & 0,02 & 0,31 & 0,17 & $-0,19$ & $-0,04$ & $-0,02$ \\
\hline Autriche & & 0,60 & 0,07 & 0,02 & 0,15 & 0,00 & 0,32 & 0,11 & $-0,20$ & $-0,07$ & $-0,03$ \\
\hline France & & 0,49 & 0,10 & 0,02 & 0,13 & 0,04 & 0,39 & 0,06 & $-0,22$ & $-0,08$ & $-0,03$ \\
\hline Allemagne & & 0,58 & 0,12 & 0,03 & 0,16 & 0,01 & 0,39 & 0,11 & $-0,27$ & $-0,08$ & $-0,07$ \\
\hline Tchéquie & & 0,50 & 0,04 & 0,04 & 0,10 & 0,01 & 0,43 & 0,10 & $-0,15$ & $-0,07$ & $-0,01$ \\
\hline Belgique & & 0,49 & 0,17 & 0,03 & 0,13 & 0,00 & 0,35 & 0,14 & $-0,31$ & $-0,10$ & $-0,05$ \\
\hline Royaume-Uni & & 0,72 & 0,05 & 0,02 & 0,13 & 0,11 & 0,48 & 0,16 & $-0,52$ & $-0,11$ & $-0,08$ \\
\hline Portugal & & 0,78 & 0,08 & 0,04 & 0,13 & 0,00 & 0,58 & 0,22 & $-0,67$ & $-0,21$ & $-0,07$ \\
\hline Italie & & 1,11 & 0,04 & 0,00 & 0,09 & 0,00 & 0,54 & 0,13 & $-0,58$ & $-0,38$ & $-0,17$ \\
\hline Grèce & & 1,18 & 0,16 & 0,06 & 0,17 & 0,06 & 0,60 & 0,12 & $-0,48$ & $-0,90$ & $-0,06$ \\
\hline Espagne & & 1,36 & 0,43 & 0,03 & 0,08 & 0,03 & 0,93 & 0,23 & $-1,41$ & $-0,67$ & $-0,10$ \\
\hline Pologne & & 1,37 & 0,11 & 0,01 & 0,13 & 0,00 & 0,51 & 0,31 & $-0,75$ & $-0,72$ & $-0,09$ \\
\hline Slovaquie & & 1,37 & 0,15 & 0,23 & 0,28 & 0,02 & 1,13 & 0,29 & $-1,42$ & $-0,99$ & $-0,10$ \\
\hline Lettonie & & 1,51 & 0,09 & 0,02 & 0,29 & 0,02 & 0,52 & 0,25 & $-1,22$ & $-0,31$ & $-0,26$ \\
\hline Estonie & & 1,53 & 0,04 & 0,08 & 0,40 & 0,02 & 1,04 & 0,30 & $-1,94$ & $-0,42$ & $-0,09$ \\
\hline Lituanie & & 1,98 & 0,08 & 0,17 & 0,36 & 0,00 & 1,24 & 0,38 & $-1,94$ & $-0,82$ & $-0,53$ \\
\hline \multicolumn{12}{|c|}{ Elasticités du dénominateur du TSD $=$ (Population en âge d'être actif) ${ }^{*}($ Revenu de référence) } \\
\hline Norvège & & 0,00 & 0,00 & 0,00 & 0,05 & 0,00 & 0,12 & 0,06 & 0,58 & 0,12 & 0,08 \\
\hline Suède & & 0,00 & 0,00 & 0,00 & 0,04 & 0,01 & 0,10 & 0,05 & 0,62 & 0,15 & 0,03 \\
\hline Pays-Bas & & 0,00 & 0,00 & 0,00 & 0,02 & 0,01 & 0,11 & 0,04 & 0,53 & 0,15 & 0,15 \\
\hline Danemark & & 0,00 & 0,00 & 0,00 & 0,05 & 0,01 & 0,07 & 0,04 & 0,66 & 0,10 & 0,06 \\
\hline Finlande & & 0,00 & 0,00 & 0,00 & 0,04 & 0,00 & 0,07 & 0,04 & 0,65 & 0,14 & 0,06 \\
\hline Autriche & & 0,00 & 0,00 & 0,00 & 0,03 & 0,00 & 0,08 & 0,03 & 0,58 & 0,20 & 0,08 \\
\hline France & & 0,00 & 0,00 & 0,00 & 0,03 & 0,01 & 0,08 & 0,01 & 0,58 & 0,22 & 0,07 \\
\hline Allemagne & & 0,00 & 0,00 & 0,00 & 0,03 & 0,00 & 0,08 & 0,02 & 0,56 & 0,16 & 0,15 \\
\hline Tchéquie & & 0,00 & 0,00 & 0,00 & 0,02 & 0,00 & 0,08 & 0,02 & 0,57 & 0,27 & 0,04 \\
\hline Belgique & & 0,00 & 0,00 & 0,00 & 0,02 & 0,00 & 0,06 & 0,02 & 0,60 & 0,19 & 0,10 \\
\hline Royaume-Uni & & 0,00 & 0,00 & 0,00 & 0,02 & 0,02 & 0,07 & 0,02 & 0,64 & 0,13 & 0,10 \\
\hline Portugal & & 0,00 & 0,00 & 0,00 & 0,02 & 0,00 & 0,07 & 0,03 & 0,62 & 0,20 & 0,07 \\
\hline Italie & & 0,00 & 0,00 & 0,00 & 0,01 & 0,00 & 0,06 & 0,01 & 0,47 & 0,31 & 0,14 \\
\hline Grèce & & 0,00 & 0,00 & 0,00 & 0,01 & 0,01 & 0,05 & 0,01 & 0,31 & 0,58 & 0,04 \\
\hline Espagne & & 0,00 & 0,00 & 0,00 & 0,00 & 0,00 & 0,05 & 0,01 & 0,60 & 0,29 & 0,04 \\
\hline Pologne & & 0,00 & 0,00 & 0,00 & 0,01 & 0,00 & 0,03 & 0,02 & 0,45 & 0,43 & 0,05 \\
\hline Slovaquie & & 0,00 & 0,00 & 0,00 & 0,01 & 0,00 & 0,06 & 0,02 & 0,52 & 0,36 & 0,03 \\
\hline Lettonie & & 0,00 & 0,00 & 0,00 & 0,02 & 0,00 & 0,03 & 0,01 & 0,64 & 0,16 & 0,14 \\
\hline Estonie & & 0,00 & 0,00 & 0,00 & 0,02 & 0,00 & 0,05 & 0,01 & 0,73 & 0,16 & 0,03 \\
\hline Lituanie & & 0,00 & 0,00 & 0,00 & 0,01 & 0,00 & 0,04 & 0,01 & 0,55 & 0,23 & 0,15 \\
\hline
\end{tabular}

Lecture : en 2002, une augmentation relative du montant des prestations retraite (col. 1) de $1 \%$ en Norvège entraîne une augmentation relative du numérateur $d u$ TSD de seulement 0,32\% du fait de sa minoration par l'augmentation de l'insécurité économique alors qu'elle n'implique aucune modification du dénominateur. En revanche, une variation relative des salaires (col. 8) de $1 \%$ en Lituanie y entraîne une diminution relative du numérateur de 1,94\% du fait de l'importance de la population pauvre en insécurité économique alors qu'elle n'implique qu'un accroissement relatif du revenu disponible moyen de 0,55\%, à savoir le dénominateur.

La connaissance de l'élasticité du numérateur (tableau 4) permet ensuite, lorsqu'on la met en rapport avec l'élasticité du TSD (tableau 2), d'évaluer sa contribution relative, et donc le rendement des différentes prestations ${ }^{24}$ dans chaque pays (tableau 3). Ainsi, toujours dans le cas de la Norvège, le tableau 3 nous indique que le rendement des prestations maladie $(0,70$ en col. 8) est égal au rapport entre l'élasticité du tableau $2(0,28)$ et celle du tableau $4(0,70)$, ce qui explique son niveau plus faible qu'en Lituanie (où le rendement est respectivement de $0,97$, soit $1,20 / 1,24)$.

\footnotetext{
${ }^{24}$ Il s'agit plus précisément du rendement des prestations net de leur contribution à l'accroissement de l'insécurité économique, laquelle est prise en compte dans le numérateur. Nous avons calculé également les rendements bruts des différentes prestations et nous pouvons les transmettre sur demande à tout lecteur intéressé. L'essentiel est de savoir qu'ils sont également constants pour toutes les prestations à vocation universelle et égaux à 1 pour les prestations destinées aux personnes sans emploi.
} 
